\title{
Approximation Methods for Inverse Problems Governed by Nonlinear Parabolic Systems*
}

\author{
H. T. Banks $\dagger$ C. J. Musante \\ Center for Research in Scientific Computation, \\ and \\ Department of Mathematics \\ North Carolina State University \\ Raleigh, NC 27695-8205
}

December 17, 1999

\begin{abstract}
We present a rigorous theoretical framework for approximation of nonlinear parabolic systems with delays in the context of inverse least squares problems. Convergence of approximate optimal parameters and that of forward solutions in the context of semidiscrete Galerkin schemes are given. Sample numerical results demonstrating the convergence are given for a model of dioxin uptake and elimination in a distributed liver model that is a special case of the general theoretical framework.
\end{abstract}

Keywords: Inverse problems, nonlinear parabolic systems, approximation techniques, least squares optimization, computational methods

${ }^{*}$ This research was supported in part by the Air Force Office of Scientific Research (AFOSR) under Grants F-49620-95-1-0236, F-49620-98-1-0180, F-49620-95-1-0375, and F-49620-93-1-0355. The research of CJM and JKR was funded by US Department of Education Graduate Assistance in Areas of National Need (GAANN) Fellowships under Grants P200A50075 and P200A70707, respectively. CJM also received support from the 1997 AFOSR Graduate Summer Research Program and the Armstrong Laboratory. Part of this research was carried out while HTB and CJM were visitors of the Institut Henri Poincaré, Centre Emile Borel.

$\dagger$ Author to whom correspondence should be addressed.

$\ddagger$ Current address: U.S. Environmental Protection Agency, National Health and Environmental Effects Research Laboratory, Experimental Toxicology Division, Mail Drop 74, Research Triangle Park, NC 27711. 


\section{Introduction}

In this paper we present results for approximation of parameter estimation problems governed by nonlinear parabolic partial differential equations with delays. Motivated by a concrete example for dioxin uptake and transport in a spatially distributed model of the liver, we formulate an inverse problem in an operator theoretic setting for a least squares optimization problem. A family of approximate optimization problems that are amenable to computation is defined in terms of least squares optimization subject to finite dimensional state space constraints. We then give convergence arguments for approximate optimal parameters to best least squares estimates for the original infinite dimensional constrained problem. To demonstrate applicability of our ideas, we point out that finite element approximations in Galerkin semi-discrete formulations based on piecewise linear spline elements satisfy all conditions of the approximation framework. Included as a special case of our optimal parameter convergence theory is the theory for convergence of numerical solutions to forward problems for nonlinear parabolic distributed parameter systems with delays. We present sample numerical results to demonstrate convergence properties in both forward simulation problems and in inverse problems with noisy data.

\section{Description and Well-Posedness of TCDD Model}

\subsection{The TCDD Model}

In this section, we present a mathematical model (1) that has been developed [1, 2] to describe pharmacokinetic and pharmacodynamic properties of TCDD. A convection-dispersion equation (1a), based on the work of Roberts and Rowland [3], characterizes the transport of blood elements in the liver sinusoidal (blood) region. Throughout this discussion, the dimensionless spatial variable $x$ takes values in the range $[0,1] ; x=0$ corresponds to the liver inlet, while $x=1$ corresponds to the outlet. Uptake of dioxin into the hepatic cells, called hepatocytes, is assumed to occur by passive diffusion. The model includes the dynamics of TCDD-binding with two intracellular hepatic proteins, the Ah receptor (1c)-(1d) and an inducible microsomal protein, CYP1A2 (1e)-(1f). The induction mechanism is described in terms of the fractional occupancy of the Ah receptor at a previous time, $t-\tau_{r}$, to account for the many intracellular processes which must occur before an increase in CYP1A2 concentration is realized. Elimination in the liver (by metabolism and biliary clearance) is assumed to be a first order process. A well-mixed, combined venous/arterial blood compartment (1g), which includes a loss due to the uptake and elimination of TCDD in the rest of the body, completes the system. A circulatory lag, $\tau_{c}$, accounts for the time delay in transport of blood elements from the exit of the liver to the venous measurement location. 
The mathematical system under consideration is as follows:

$$
\begin{aligned}
& \left(V_{B}+V_{D} \frac{f_{u_{B}}}{f_{u_{D}}}\right) \frac{\partial C_{B}}{\partial t}=Q \mathcal{D}_{N} \frac{\partial^{2} C_{B}}{\partial x^{2}}-Q \frac{\partial C_{B}}{\partial x}+P\left(C_{u_{H}}-f_{u_{B}} C_{B}\right), \\
& \frac{\partial C_{u_{H}}}{\partial t}=\frac{P f_{u_{B}}}{V_{H}} C_{B}-\left(\frac{P}{V_{H}}+k_{3}\right) C_{u_{H}}-g_{A h}\left(C_{u_{H}}, C_{A h}\right) \\
& +k_{-1} C_{A h-T}-g_{P r}\left(C_{u_{H}}, C_{P r}\right)+k_{-2} C_{P r-T}, \\
& \frac{\partial C_{A h-T}}{\partial t}=g_{A h}\left(C_{u_{H}}, C_{A h}\right)-k_{-1} C_{A h-T}, \\
& \frac{\partial C_{A h}}{\partial t}=k_{-1} C_{A h-T}-g_{A h}\left(C_{u_{H}}, C_{A h}\right)-k_{d(A h)} C_{A h}+k_{s(A h)}, \\
& \frac{\partial C_{P r-T}}{\partial t}=g_{P r}\left(C_{u_{H}}, C_{P r}\right)-k_{-2} C_{P r-T}, \\
& \frac{\partial C_{P r}}{\partial t}=k_{-2} C_{P r-T}-g_{P r}\left(C_{u_{H}}, C_{P r}\right)-k_{d(P r)} C_{P r}+k_{s(P r)} \\
& +I_{P r} \frac{C_{A h-T}\left(t-\tau_{r}\right)}{C_{A h}\left(t-\tau_{r}\right)+C_{A h-T}\left(t-\tau_{r}\right)}, \\
& \frac{d C_{a}}{d t}(t)=\frac{Q_{a}}{V_{a}}\left(C_{B}\left(t-\tau_{c}, 1\right)-C_{a}(t)\right)+I(t)-k_{e} C_{a}(t), \\
& C_{B}(t, 0)=C_{a}(t), \\
& Q C_{B}(t, 1)-Q \mathcal{D}_{N} \frac{\partial C_{B}}{\partial x}(t, 1)=A q_{2}(t), \\
& C(s, x)=\Phi(x), \quad s \in\left[-\tau_{r}, 0\right] \text {, }
\end{aligned}
$$

where $C=\left[C_{B}, C_{u_{H}}, C_{A h-T}, C_{A h}, C_{P r-T}, C_{P r}, C_{a}\right]^{T}$. In (1h), $\Phi$ and $A q_{2}$ are assumed known.

We remark that $g_{A h}$ and $g_{P r}$ in equations (1b)-(1f) are saturating nonlinearities (see [2]) modified from the usual product terms,

$$
\begin{aligned}
& \tilde{g}_{A h}(y, z)=k_{+1} y z, \\
& \tilde{g}_{P r}(y, z)=k_{+2} y z, \quad \text { for } y, z \in \mathbb{R},
\end{aligned}
$$

arising from the law of mass action in chemical kinetics. Specifically, we assume that within a certain range of concentrations the system behaves according to the nonlinearities prescribed by (2) and (3) but eventually saturates; i.e., due to the availability of binding species, we assume the rates of formation of Ah-TCDD complex and CYP1A2-TCDD complex are bounded.

The summary given above, while brief, is included to provide the reader with a general understanding of the complex dynamics of the system under investigation. The reader is referred to the aforementioned works $[1,2,4]$ for complete discussions. 


\subsection{Well-Posedness}

The mathematical model consists of a nonlinear system of partial differential equations with delays and questions of well-posedness are of interest. A detailed general theory of existence, uniqueness, and continuous dependence for systems which include the model above is given in $[2,4]$. The results, summarized below, were obtained using a weak or variational formulation of the problem statement and are based on ideas from the work of Banks et al. [5] for nonlinear hyperbolic systems.

The initial-boundary-value problem (1) can be restated in terms of a weak or variational formulation (see $[2,4]$ for details). In particular, we consider the state space $\mathcal{V}=$ $V \times H^{5} \times \mathbb{R}$ and $\mathcal{H}=H^{6} \times \mathbb{R}$, where $V=H_{L}^{1}(0,1)$ and $H=L_{2}(0,1)$. We define

$$
H_{L}^{1}(0,1)=\left\{\phi \in H^{1}(0,1) \mid \phi(0)=0\right\},
$$

with $V$-norm

$$
|\phi|_{V}=\left|\phi^{\prime}\right|_{H}, \text { for all } \phi \in V .
$$

The inner product in $\mathcal{H}$ is defined by

$$
\langle\phi, \psi\rangle_{\mathcal{H}}=\sum_{k=1}^{6}\left\langle\phi_{k}, \psi_{k}\right\rangle+\phi_{7} \psi_{7} \quad \text { for all } \phi, \psi \in \mathcal{H},
$$

where $\langle\cdot, \cdot\rangle$ denotes the usual $L_{2}$ inner product:

$$
\langle f, g\rangle=\int_{0}^{1} f(\xi) g(\xi) d \xi .
$$

We multiply the $i^{\text {th }}$ equation in (1) by a function $\phi_{i}$ in a "suitable" class of test functions and integrate in space in the first six equations, followed by integration by parts in the first equation (1a) only. The weak or variational formulation of the problem (1) can be written in operator theoretic form - see $[2,4]$ for precise definitions of the operators and nonlinear functions - as follows: we seek a solution $y(t) \in \mathcal{V}$ satisfying

$$
\begin{gathered}
\dot{y}(t)+\mathcal{A} y(t)+\mathcal{A}_{D}\left(y\left(t-\tau_{c}\right)\right)+g(y(t))+g_{D}\left(y\left(t-\tau_{r}\right)\right)=F(t) \text { in } \mathcal{V}^{*}, \\
y(s)=y_{0}(s), \quad s \in[-\tau, 0] .
\end{gathered}
$$

Theorem 1 Under certain mild assumptions (see [2, 4]) on the problem data, there exists a weak solution $y$ of (4) with $y \in L_{2}((0, T), \mathcal{V}) \cap C([0, T], \mathcal{H})$ and $\dot{y} \in L_{2}\left((0, T), \mathcal{V}^{*}\right)$. Furthermore, the solution is unique and depends continuously on the data $\left(y_{0}, F\right)$.

This result was obtained by first establishing well-posedness for the system

$$
\begin{gathered}
\dot{y}(t)+\mathcal{A} y(t)+g(y(t))=F(t) \text { in } \mathcal{V}^{*}, \\
y(s)=y_{0},
\end{gathered}
$$


and then showing that the result could be extended to delay systems of the form (4) by the method of steps [6] used in the study of delay differential equations. It was thus noted that the system (1), or more generally, (4), could be theoretically and conceptually treated by investigations of the abstract system (5).

\section{Parameter Estimation and Convergence of Galerkin Approximations}

The mathematical model summarized in Section 2.1 contains a number of physiological, biological, and modeling parameters, including permeability coefficients, rate constants, flow rates, and the dispersion coefficient. If this model is to be used for simulation of the distribution and elimination of TCDD in animals, values for these parameters must be known. Although some values can be measured explicitly, others must be estimated from experimental data. Thus, a method to estimate the so-called "unknown" parameters is needed.

Based on the theoretical development described in Section 2.2, and since the model (1) is a special case of (4), which can be investigated via (5), we consider the following class of abstract nonlinear parameter dependent parabolic systems evolving in a real separable Hilbert space:

$$
\begin{gathered}
\dot{y}(t)+\mathcal{A}(q) y(t)+g(q)(y(t))=F(t ; q) \\
y(0)=y_{0} .
\end{gathered}
$$

In this case the unbounded operator $\mathcal{A}$, the nonlinear operator $g$, and the forcing term $F$ are all assumed to be dependent on some parameter $q$, in contrast to the "parameter free" representation given in $(5)$.

The results presented here are based on the general parameter estimation formulation and results of Banks and Kunisch [7]. In this general estimation formulation of the system in (5), we consider (6) where the linear operator $\mathcal{A}$, the nonlinear term $g$, and the input $F$ have all been parameterized by a vector parameter $q$ that must be estimated from experimental data. In this case $q$ takes on values from an admissible parameter set $Q$, which may be an infinite dimensional set. Given a data set of observations $w=\left\{w_{i}\right\}_{i=1}^{K}$ corresponding to measurements taken at time $t_{i}$, we seek to solve the general least squares parameter estimation problem

$$
\min _{q \in Q} J(q, w)=\sum_{i=1}^{K}\left|C y\left(t_{i}, \cdot ; q\right)-w_{i}\right|^{2},
$$

where $\left\{y\left(t_{i}, \cdot ; q\right)\right\}$ are the parameter dependent solutions of $(6)$ and $|\cdot|$ is an appropriately chosen Euclidean norm. The observation operator $C$ may take many different forms (see [7]) 
depending on the type of data collected. For example, if $w_{i}$ is the concentration at a point $z$ and time $t_{i}$, then $C$ involves function evaluation in space.

This minimization problem involves an infinite dimensional state space $\mathcal{H}$ and an admissible parameter set $Q$. The parameter space is generally infinite dimensional as certain of the unknown parameters (for example, the exit flux $q_{2}(t)$ discussed in Section 2) involve spatial and/or temporal dependence. We proceed as in the works of Banks et al. [7, 8, 9]. Let $\mathcal{H}^{N}$ be a finite dimensional subspace of $\mathcal{H}$ and $Q^{M}$ be a sequence of finite dimensional sets approximating $Q$. We then can formulate a family of finite dimensional estimation problems, with finite dimensional state spaces and finite dimensional parameter sets, as follows: find $q \in Q^{M}$ which minimizes

$$
J^{N}(q, w)=\left|C y^{N}\left(t_{i}, \cdot ; q\right)-w_{i}\right|^{2},
$$

where $y^{N}(t ; q) \in \mathcal{H}^{N}$ is the solution to the finite dimensional approximation of (6) given by:

$$
\begin{gathered}
\left\langle\dot{y}^{N}, \phi\right\rangle_{\mathcal{V}^{*}, \mathcal{V}}+\left\langle\mathcal{A}(q) y^{N}, \phi\right\rangle_{\mathcal{V}^{*}, \mathcal{V}}+\left\langle g(q)\left(y^{N}\right), \phi\right\rangle=\langle F(t ; q), \phi\rangle_{\mathcal{V}^{*}, \mathcal{V}} \\
y^{N}(0)=P^{N} y_{0},
\end{gathered}
$$

for all $\phi \in \mathcal{H}^{N}$, where $P^{N}$ is the orthogonal projection of $\mathcal{H}$ onto $\mathcal{H}^{N}$.

A sequence of parameter estimates $\left\{\bar{q}^{N, M}\right\}$ results from the solution of these approximate estimation problems (7)-(8). The question we address in this section is when one can guarantee that this sequence converges to a solution of the original infinite dimensional parameter estimation problem. A general unifying framework for least-squares minimization problems for first and second order systems has been presented in [10]. Conditions guaranteeing convergence for hyperbolic problems have been presented in [9] for linear systems and were extended in [8] to treat a nonlinear case. We adapt these results to a certain class of nonlinear parabolic systems which includes the TCDD model.

In Section 2.2 we outlined general well-posedness results which confirmed conditions under which the system (5) (or (6) without considering the parameter dependence) has a unique weak solution. In this section we state precise conditions under which (6), as well as the finite dimensional problem (8), have a unique weak solution for each $q \in Q$. We also detail the assumptions on the general parameter identification problem which are used in the convergence result, and state an abstract sufficient condition, given in [9], for the convergence of the sequence of solutions $\left\{\bar{q}^{N, M}\right\}$ of the finite dimensional estimation problems to a solution of the original infinite dimensional problem. Finally, we show that these conditions are satisfied for a general class of nonlinear parabolic systems which includes the TCDD model. 


\subsection{Formulation of the Problem}

We assume the general state space setting detailed in [2]; that is, there is a sequence of real separable Hilbert spaces $\mathcal{V}, \mathcal{H}, \mathcal{V}^{*}$ forming a Gelfand triple [11] satisfying

$$
\mathcal{V} \hookrightarrow \mathcal{H} \simeq \mathcal{H}^{*} \hookrightarrow \mathcal{V}^{*}
$$

where we assume that the embedding $\mathcal{V} \hookrightarrow \mathcal{H}$ is dense and continuous with

$$
|\phi|_{\mathcal{H}} \leq k|\phi|_{\mathcal{V}} \text { for } \phi \in \mathcal{V}
$$

We denote by $\langle\cdot, \cdot\rangle_{\mathcal{V}^{*}, \mathcal{V}}$ the usual duality product $[9,11]$, which is the extension by continuity of the inner product in $\mathcal{H}$, denoted $\langle\cdot, \cdot\rangle$. The norm in $\mathcal{H}$ will be denoted $|\cdot|$. The operator $\mathcal{A}(q)$ is defined (under the assumptions below) in terms of an associated sesquilinear form $\sigma(q): \mathcal{V} \times \mathcal{V} \rightarrow \mathbb{R}$; that is, $\mathcal{A}(q) \in \mathcal{L}\left(\mathcal{V}, \mathcal{V}^{*}\right)$ and $\langle\mathcal{A}(q) \phi, \psi\rangle_{\mathcal{V}^{*}, \mathcal{V}}=\sigma(q)(\phi, \psi)$

We make the following standing assumptions to establish our parameter estimation convergence results. It should be noted that these assumptions are the same as those presented in [2] (however in [2] they are denoted by $\left.\left.\mathrm{A} 1^{\prime}\right)-\mathrm{A} 6^{\prime}\right)$ ), except that we now require them to be satisfied uniformly for all $q \in Q$. Thus, well-posedness of a weak solution is guaranteed, under the conditions of Theorem 1 stated in Section 2 and Theorem 2.2.1 in [2], for all $q \in Q$. Our standing assumptions are:

A1) The form $\sigma(q)$ is $\mathcal{V}$-bounded: for all $\phi, \psi \in \mathcal{V}$ there exists a positive constant $\gamma_{1}$ (independent of $q$ ) such that

$$
|\sigma(q)(\phi, \psi)| \leq \gamma_{1}|\phi|_{\mathcal{V}}|\psi|_{\mathcal{V}}
$$

for every $q \in Q$.

A2) The form $\sigma(q)$ is strictly coercive on $\mathcal{V}$ : for all $\phi \in \mathcal{V}$ there exists a positive constant $k_{1}$ (independent of q) such that

$$
\sigma(q)(\phi, \phi) \geq k_{1}|\phi|_{\mathcal{V}}^{2}
$$

for all $q \in Q$.

A3) The forcing term $F(\cdot ; q)$ satisfies

$$
F(\cdot ; q) \in L_{2}\left((0, T), \mathcal{V}^{*}\right)
$$

for every $q \in Q$. 
A4) The function $g(q)$ is a continuous nonlinear mapping from $\mathcal{H}$ into $\mathcal{H}$ satisfying

$$
|g(q)(\phi)| \leq \gamma|\phi|, \quad \phi \in \mathcal{H}
$$

for some positive constant $\gamma$ and for every $q \in Q$.

A5) For any $\phi, \psi \in \mathcal{V}$,

$$
\langle g(q)(\phi)-g(q)(\psi), \phi-\psi\rangle+k_{1} k^{-1}|\phi-\psi|^{2} \geq 0
$$

where $k$ and $k_{1}$ are the constants in (9) and (11).

A6) For any $\phi \in \mathcal{H}$ the Fréchet derivative of $g$ exists and satisfies

$$
g^{\prime}(q)(\phi) \in \mathcal{L}(\mathcal{H}, \mathcal{H}) \text { with }\left|g^{\prime}(q)(\phi)\right|_{\mathcal{L}(\mathcal{H}, \mathcal{H})} \leq \tilde{C}_{3}
$$

for every $q \in Q$.

The proofs of the following theorems can be found in [2] and [4].

Theorem 2 Under conditions A1)-A6) the system (6) has a unique weak solution $y \in$ $L_{2}((0, T), \mathcal{H})$ for every $y_{0} \in \mathcal{H}$. The weak solution depends continuously on the initial data and satisfies

$$
\langle\dot{y}(t), \phi\rangle_{\mathcal{V}^{*}, \mathcal{V}}+\sigma(q)(y(t), \phi)+\langle g(q)(y(t)), \phi\rangle=\langle F(t ; q), \phi\rangle_{\mathcal{V}^{*}, \mathcal{V}}
$$

for all $\phi \in \mathcal{V}$ and almost all $t \in(0, T)$. Moreover, $\dot{y} \in L_{2}\left((0, T), \mathcal{V}^{*}\right)$.

For the TCDD model, the convexity condition A5) on the nonlinearity $g(q)$ is not satisfied. Weakening this condition on $g$, we established well-posedness for the TCDD model by requiring greater regularity on the forcing term $F$.

Theorem 3 Suppose $\mathcal{V}$ embeds in $\mathcal{H}$ compactly. Let $F_{1}(\cdot ; q) \in L_{2}((0, T), \mathcal{H})$ and $F_{2}(\cdot ; q) \in$ $H^{1}\left((0, T), \mathcal{V}^{*}\right)$. Under assumptions A1), A2), A4), and A6) with $y_{0} \in \mathcal{V}$ and $F=F_{1}+$ $F_{2}$, there exists a weak solution $y$ of $(6)$ with $y \in L_{2}((0, T), \mathcal{V}) \cap C([0, T], \mathcal{H})$ and $\dot{y} \in$ $L_{2}\left((0, T), \mathcal{V}^{*}\right)$. Furthermore, the solution is unique and depends continuously on the data $\left(y_{0}, F\right)$. 


\subsection{The General Parameter Estimation Problem}

We turn to the least squares minimization problem described previously: find $q \in Q$ which minimizes

$$
J(q, w)=\sum_{i=1}^{K}\left|C y\left(t_{i}, \cdot ; q\right)-w_{i}\right|^{2},
$$

where $\left\{y\left(t_{i}, \cdot ; q\right)\right\}$ are the parameter dependent solutions of (6) evaluated at time $t_{i}$ and $w=\left\{w_{i}\right\}_{i=1}^{K}$ corresponds to measurements taken at time $t_{i}$. The operator $C$ is a continuous linear operator from its domain $\mathcal{F}(J, \mathcal{H})$ to the observation space $\mathcal{W}$, where $J$ is a Borel measurable subset of the maximal interval of observations $(0, \tilde{T}]$. For example, if we have discrete-discrete observations

$$
w=\left\{w\left(t_{i}, x_{j}\right)\right\}_{i=1, \ldots, r}^{j=1, \ldots, l} \in \mathcal{W}=\mathbb{R}^{r \times l},
$$

then

$$
C \psi=\left\{\psi\left(t_{i}, x_{j}\right)\right\}_{i=1, \ldots, r}^{j=1, \ldots, l}
$$

where the natural choice for the function space $\mathcal{F}(J, \mathcal{H})$ is $C(J, C(0,1))$. We once again consider Galerkin type approximations to (6) and, as discussed in the introduction to this section, define a family of approximating parameter estimation problems.

The approximate parameter identification problems we consider entail minimization of $(7)$. As in $[8,9]$, we make the following assumptions for the infinite dimensional spaces $\mathcal{H}, Q$ and for the finite dimensional subspaces $\mathcal{H}^{N}, Q^{M}$ :

B1) The sets $Q$ and $Q^{M}$ lie in a metric space $\tilde{Q}$ with metric $d$. The subspaces $Q$ and $Q^{M}$ are compact in this metric and there exists a mapping $i^{M}: Q \rightarrow Q^{M}$ such that $Q^{M}=i^{M}(Q)$. Furthermore, for each $q \in Q, i^{M}(q) \rightarrow q$ in $\tilde{Q}$ with the convergence uniform in $q \in Q$.

B2) The finite dimensional subspaces $\mathcal{H}^{N}$ satisfy $\mathcal{H}^{N} \subset \mathcal{V}$.

B3) For each $\psi \in \mathcal{V},\left|\psi-P^{N} \psi\right|_{\mathcal{V}} \rightarrow 0$ as $N \rightarrow \infty$.

We further assume that the operators $\mathcal{A}(q)$ and $g(q)$ as well as the forcing term $F$ depend continuously on $q \in Q$; that is, we assume that the following conditions are satisfied:

C1) $|\sigma(q)(\phi, \psi)-\sigma(\tilde{q})(\phi, \psi)| \leq d_{1}(q, \tilde{q})|\phi| \mathcal{V}|\psi|_{\mathcal{V}}$, for every $\phi, \psi \in \mathcal{V}$, where $d_{1}(q, \tilde{q}) \rightarrow 0$ as $d(q, \tilde{q}) \rightarrow 0$.

C2) $|g(q)(\phi)-g(\tilde{q})(\phi)| \leq d_{2}(q, \tilde{q})|\phi|$, for all $\phi \in \mathcal{H}$, where $d_{2}(q, \tilde{q}) \rightarrow 0$ as $d(q, \tilde{q}) \rightarrow 0$.

C3) The mapping $q \rightarrow F(\cdot ; q)$ is continuous from $Q$ to $L_{2}\left((0, T), \mathcal{V}^{*}\right)$. 
Under the conditions of Theorem 2 or Theorem 3 and C1)-C3), the theory of parameter dependence of solutions to ordinary differential equations [12, Theorem 6.3.1] guarantees that the mapping $q \rightarrow y^{N}(t ; q)$ is continuous for each $t$. Furthermore, under condition B1) we know that a solution $\left\{\bar{q}^{N, M}\right\}$ to the approximate parameter identification problem (7)-(8) exists. A general sufficient condition for the convergence of $\left\{\bar{q}^{N, M}\right\}$ to $\bar{q}$, a solution to the infinite dimensional minimization problem, is given in Theorem 5.1 of [9]:

Theorem 4 To obtain convergence of at least a subsequence of $\left\{\bar{q}^{N, M}\right\}$ to a solution $\bar{q}$ minimizing (17) subject to (6), it suffices, under assumption B1), to argue that for arbitrary sequences $\left\{q^{N, M}\right\}$ in $Q^{M}$ with $q^{N, M} \rightarrow q \in Q$, we have

$$
C y^{N}\left(t ; q^{N, M}\right) \rightarrow C y(t ; q) .
$$

Note that condition (18) involves convergence of the original sequence of Galerkin approximations, which is a stronger result than the subsequential convergence obtained in Chapter 2 of [2] and a computationally important distinction.

\subsection{Convergence Results}

We show in this section that the convergence criterion of Theorem 4 holds under the general conditions stated above. Without loss of generality, we suppress the double index notation on the parameter $q^{N, M}$. This is possible due to the assumptions in B1) - see [7, 9].

Theorem 5 Assume that the conditions of Theorem 3 are satisfied, along with B1)-B3) and C1)-C3). Let $q^{N}$ be any sequence in $Q^{N}$ such that $q^{N} \rightarrow q \in Q$. Then

$$
y^{N}\left(t ; q^{N}\right) \rightarrow y(t ; q) \text { in } \mathcal{H}
$$

uniformly on $[0, T]$, and

$$
y^{N}\left(t ; q^{N}\right) \rightarrow y(t ; q) \text { in } \mathcal{V}
$$

for almost all $t>0$, where $y^{N}$ satisfies

$$
\begin{gathered}
\left\langle\dot{y}^{N}(t), \phi\right\rangle_{\mathcal{V}^{*}, \mathcal{V}}+\sigma\left(q^{N}\right)\left(y^{N}(t), \phi\right)+\left\langle g\left(q^{N}\right)\left(y^{N}(t)\right), \phi\right\rangle=\left\langle F\left(t ; q^{N}\right), \phi\right\rangle_{\mathcal{V}^{*}, \mathcal{V}} \\
y^{N}(0)=P^{N} y_{0},
\end{gathered}
$$

for all $\phi \in \mathcal{H}^{N}$, and y satisfies

$$
\begin{gathered}
\langle\dot{y}(t), \phi\rangle_{\mathcal{V}^{*}, \mathcal{V}}+\sigma(q)(y(t), \phi)+\langle g(q)(y(t)), \phi\rangle=\langle F(t ; q), \phi\rangle_{\mathcal{V}^{*}, \mathcal{V}} \\
y(0)=y_{0},
\end{gathered}
$$

for all $\phi \in \mathcal{V}$. 
Proof: We note, by the results of Theorem 3 , that $y(t) \in \mathcal{H}$ for all $t \geq 0$ and $y(t) \in \mathcal{V}$ almost everywhere. By the triangle inequality, we obtain

$$
\left|y^{N}\left(t ; q^{N}\right)-y(t ; q)\right| \leq\left|y^{N}\left(t ; q^{N}\right)-P^{N} y(t ; q)\right|+\left|P^{N} y(t ; q)-y(t ; q)\right|
$$

for each $t \in[0, T]$. Assumption B3) states that for each $\psi \in \mathcal{V},\left|\psi-P^{N} \psi\right|_{\mathcal{V}} \rightarrow 0$ as $N \rightarrow \infty$, which of course implies $\left|\psi-P^{N} \psi\right|_{\mathcal{H}} \rightarrow 0$ for $\phi \in \mathcal{H}$. Since $\{y(t): t \in[0, T]\}$ is compact in $\mathcal{H}$, the convergence

$$
\left|P^{N} y(t ; q)-y(t ; q)\right|_{\mathcal{H}} \rightarrow 0
$$

is uniform on $[0, T]$. Hence, we need only show

$$
\left|y^{N}\left(t ; q^{N}\right)-P^{N} y(t ; q)\right|_{\mathcal{H}} \rightarrow 0 \text { as } N \rightarrow \infty
$$

uniformly on $[0, T]$ to obtain the desired convergence in $\mathcal{H}$. The desired convergence in $\mathcal{V}$ follows in the same manner from (21) by arguing

$$
\left|y^{N}\left(t, q^{N}\right)-P^{N} y(t, q)\right|_{\mathcal{V}} \rightarrow 0 \text { as } N \rightarrow \infty
$$

for almost every $t>0$.

Proceeding as in [8], we define

$$
\begin{aligned}
y^{N} & =y^{N}\left(t ; q^{N}\right), \\
y & =y(t ; q), \\
\Delta^{N} & =y^{N}\left(t ; q^{N}\right)-P^{N} y(t ; q)=y^{N}-P^{N} y,
\end{aligned}
$$

from which it follows that

$$
\begin{aligned}
\Delta_{t}^{N} & =y_{t}^{N}-\frac{d}{d t} P^{N} y \\
& =y_{t}^{N}-P^{N} y_{t} .
\end{aligned}
$$

Note for all $\phi \in \mathcal{H}^{N}$,

$$
\begin{aligned}
\left\langle\Delta_{t}^{N}, \phi\right\rangle_{\mathcal{V}^{*}, \mathcal{V}} & =\left\langle y_{t}^{N}-\frac{d}{d t} P^{N} y, \phi\right\rangle_{\mathcal{V}^{*}, \mathcal{V}} \\
& =\left\langle y_{t}^{N}-y_{t}+y_{t}-\frac{d}{d t} P^{N} y, \phi\right\rangle_{\mathcal{V}^{*}, \mathcal{V}} \\
& =\left\langle y_{t}^{N}, \phi\right\rangle_{\mathcal{V}^{*}, \mathcal{V}}-\left\langle y_{t}, \phi\right\rangle_{\mathcal{V}^{*}, \mathcal{V}}+\left\langle y_{t}-\frac{d}{d t} P^{N} y, \phi\right\rangle_{\mathcal{V}^{*}, \mathcal{V}}
\end{aligned}
$$

Now by (19a) and (20a) we have,

$$
\left\langle\Delta_{t}^{N}, \phi\right\rangle_{\mathcal{V}^{*}, \mathcal{V}}=\left\langle F\left(t ; q^{N}\right), \phi\right\rangle_{\mathcal{V}^{*}, \mathcal{V}}-\sigma\left(q^{N}\right)\left(y^{N}, \phi\right)-\left\langle g\left(q^{N}\right)\left(y^{N}\right), \phi\right\rangle
$$




$$
\begin{aligned}
& -\langle F(t ; q), \phi\rangle_{\mathcal{V}^{*}, \mathcal{V}}+\sigma(q)(y, \phi)+\langle g(q)(y), \phi\rangle \\
& +\left\langle y_{t}-\frac{d}{d t} P^{N} y, \phi\right\rangle_{\mathcal{V}^{*}, \mathcal{V}} \\
= & \left\langle y_{t}-\frac{d}{d t} P^{N} y, \phi\right\rangle_{\mathcal{V}^{*}, \mathcal{V}}+\left\langle F\left(t ; q^{N}\right)-F(t ; q), \phi\right\rangle_{\mathcal{V}^{*}, \mathcal{V}} \\
& -\sigma\left(q^{N}\right)\left(y^{N}, \phi\right)+\sigma(q)(y, \phi)+\sigma\left(q^{N}\right)\left(y-P^{N} y, \phi\right) \\
& -\sigma\left(q^{N}\right)\left(y-P^{N} y, \phi\right)+\left\langle g(q)(y)-g\left(q^{N}\right)\left(y^{N}\right), \phi\right\rangle, \\
= & \left\langle y_{t}-\frac{d}{d t} P^{N} y, \phi\right\rangle_{\mathcal{V}^{*}, \mathcal{V}}+\left\langle F\left(t ; q^{N}\right)-F(t ; q), \phi\right\rangle_{\mathcal{V}^{*}, \mathcal{V}} \\
& -\sigma\left(q^{N}\right)\left(y^{N}-P^{N} y, \phi\right)+\sigma(q)(y, \phi)-\sigma\left(q^{N}\right)(y, \phi) \\
& +\sigma\left(q^{N}\right)\left(y-P^{N} y, \phi\right)+\left\langle g(q)(y)-g\left(q^{N}\right)\left(y^{N}\right), \phi\right\rangle,
\end{aligned}
$$

by adding and subtracting $\sigma\left(q^{N}\right)\left(y-P^{N} y, \phi\right)$. Rearranging terms and using the definition of $\Delta^{N}$ we obtain the equation

$$
\begin{aligned}
\left\langle\Delta_{t}^{N}, \phi\right\rangle_{\mathcal{V}^{*}, \mathcal{V}}+\sigma\left(q^{N}\right)\left(\Delta^{N}, \phi\right)= & \left\langle y_{t}-\frac{d}{d t} P^{N} y, \phi\right\rangle_{\mathcal{V}^{*}, \mathcal{V}}+\left\langle F\left(t ; q^{N}\right)-F(t ; q), \phi\right\rangle_{\mathcal{V}^{*}, \mathcal{V}} \\
& +\sigma(q)(y, \phi)-\sigma\left(q^{N}\right)(y, \phi)+\sigma\left(q^{N}\right)\left(y-P^{N} y, \phi\right) \\
& +\left\langle g(q)(y)-g\left(q^{N}\right)\left(y^{N}\right), \phi\right\rangle,
\end{aligned}
$$

which holds for all $\phi \in \mathcal{H}^{N}$. Now, choosing $\phi=\Delta^{N} \in \mathcal{H}^{N}$ and recalling that $\left\langle\Delta_{t}^{N}, \Delta^{N}\right\rangle_{\mathcal{V}^{*}, \mathcal{V}}=$ $\frac{1}{2} \frac{d}{d t}\left|\Delta^{N}\right|^{2}$, we have

$$
\begin{aligned}
\frac{1}{2} \frac{d}{d t}\left|\Delta^{N}\right|^{2}+\sigma\left(q^{N}\right) & \left(\Delta^{N}, \Delta^{N}\right)=\left\langle y_{t}-\frac{d}{d t} P^{N} y, \Delta^{N}\right\rangle_{\mathcal{V}^{*}, \mathcal{V}} \\
+ & \left\langle F\left(t ; q^{N}\right)-F(t ; q), \Delta^{N}\right\rangle_{\mathcal{V}^{*}, \mathcal{V}}+\sigma(q)\left(y, \Delta^{N}\right) \\
& -\sigma\left(q^{N}\right)\left(y, \Delta^{N}\right)+\sigma\left(q^{N}\right)\left(y-P^{N} y, \Delta^{N}\right) \\
& +\left\langle g(q)(y)-g\left(q^{N}\right)\left(y^{N}\right), \Delta^{N}\right\rangle .
\end{aligned}
$$

Let $L(t)$ represent the left side of equation $(27)$,

$$
L(t)=\frac{1}{2} \frac{d}{d t}\left|\Delta^{N}(t)\right|^{2}+\sigma\left(q^{N}\right)\left(\Delta^{N}(t), \Delta^{N}(t)\right),
$$

and $R(t)$ represent the right side,

$$
\begin{aligned}
R(t)= & \left\langle y_{t}(t)-\frac{d}{d t} P^{N} y(t), \Delta^{N}(t)\right\rangle_{\mathcal{V}^{*}, \mathcal{V}}+\left\langle F\left(t ; q^{N}\right)-F(t ; q), \Delta^{N}(t)\right\rangle_{\mathcal{V}^{*}, \mathcal{V}} \\
& +\sigma(q)\left(y(t), \Delta^{N}(t)\right)-\sigma\left(q^{N}\right)\left(y(t), \Delta^{N}(t)\right)+\sigma\left(q^{N}\right)\left(y(t)-P^{N} y(t), \Delta^{N}(t)\right) \\
& +\left\langle g(q)(y(t))-g\left(q^{N}\right)\left(y^{N}(t)\right), \Delta^{N}(t)\right\rangle,
\end{aligned}
$$

which hold almost everywhere. Integrating $L$ from 0 to $t$ and using A2) and the initial condition

$$
\Delta^{N}(0)=y^{N}(0)-P^{N} y(0)=y^{N}(0)-P^{N} y_{0}=0,
$$


we obtain the inequality

$$
\begin{aligned}
\int_{0}^{t} L(s) d s & =\int_{0}^{t}\left\{\frac{1}{2} \frac{d}{d s}\left|\Delta^{N}(s)\right|^{2}+\sigma\left(q^{N}\right)\left(\Delta^{N}(s), \Delta^{N}(s)\right)\right\} d s \\
& \geq \frac{1}{2}\left|\Delta^{N}(t)\right|^{2}+k_{1} \int_{0}^{t}\left|\Delta^{N}(s)\right|_{\mathcal{V}}^{2} d s
\end{aligned}
$$

for all $t \geq 0$.

We next integrate $R$ from 0 to $t$ :

$$
\begin{aligned}
& \int_{0}^{t} R(s) d s \\
& =\int_{0}^{t}\left\{\left\langle y_{s}(s)-\frac{d}{d s} P^{N} y(s), \Delta^{N}(s)\right\rangle_{\mathcal{V}^{*}, \mathcal{V}}+\left\langle F\left(s ; q^{N}\right)-F(s ; q), \Delta^{N}(s)\right\rangle_{\mathcal{V}^{*}, \mathcal{V}}\right. \\
& \quad+\sigma(q)\left(y(s), \Delta^{N}(s)\right)-\sigma\left(q^{N}\right)\left(y(s), \Delta^{N}(s)\right)+\sigma\left(q^{N}\right)\left(y(s)-P^{N} y(s), \Delta^{N}(s)\right) \\
& \left.\quad+\left\langle g(q)(y(s))-g\left(q^{N}\right)\left(y^{N}(s)\right), \Delta^{N}(s)\right\rangle\right\} d s .
\end{aligned}
$$

The first term under the integral on the right side of equation (28) is identically zero. To see this, note that for any $\phi \in \mathcal{H}^{N}$,

$$
\begin{aligned}
\left\langle y_{t}-\frac{d}{d t} P^{N} y, \phi\right\rangle_{\mathcal{V}^{*}, \mathcal{V}} & =\left\langle\frac{d}{d t}\left(y-P^{N} y\right), \phi\right\rangle_{\mathcal{V}^{*}, \mathcal{V}} \\
& =\frac{d}{d t}\left\langle y-P^{N} y, \phi\right\rangle_{\mathcal{V}^{*}, \mathcal{V}} \\
& =\frac{d}{d t}\left\langle y-P^{N} y, \phi\right\rangle_{\mathcal{H}}
\end{aligned}
$$

since $y-P^{N} y \in \mathcal{H}$. Recall that $P^{N}$ is the orthogonal projection of $\mathcal{H}$ onto $\mathcal{H}^{N}$, so that $\left(I-P^{N}\right) y$ is orthogonal to elements in $\mathcal{H}^{N}$. Therefore, $\frac{d}{d t}\left\langle\left(y-P^{N} y\right), \phi\right\rangle_{\mathcal{H}} \equiv 0$ for any $\phi \in \mathcal{H}^{N}$.

To treat the second term under the integral, we use the inequality $2 a b \leq a^{2}+b^{2}$ :

$$
\begin{aligned}
\int_{0}^{t}\left\langle F\left(s ; q^{N}\right)-F(s ; q), \Delta^{N}(s)\right\rangle_{\mathcal{V}^{*}, \mathcal{V}} d s \leq & \frac{1}{2 \epsilon} \int_{0}^{t}\left|F\left(s ; q^{N}\right)-F(s ; q)\right|_{\mathcal{V}^{*}}^{2} d s \\
& +\frac{\epsilon}{2} \int_{0}^{t}\left|\Delta^{N}(s)\right|_{\mathcal{V}}^{2} d s
\end{aligned}
$$

which holds for any $\epsilon>0$.

For the third and fourth terms, we use Assumption C1) and the same technique as in the previous argument to obtain

$$
\int_{0}^{t}\left\{\sigma(q)\left(y(s), \Delta^{N}(s)\right)-\sigma\left(q^{N}\right)\left(y(s), \Delta^{N}(s)\right)\right\} d s
$$




$$
\begin{aligned}
& \leq \int_{0}^{t}\left|\sigma(q)\left(y(s), \Delta^{N}(s)\right)-\sigma\left(q^{N}\right)\left(y(s), \Delta^{N}(s)\right)\right| d s \\
& \leq \int_{0}^{t} d_{1}\left(q, q^{N}\right)|y(s)|_{\mathcal{V}}\left|\Delta^{N}(s)\right|_{\mathcal{V}} d s \\
& \leq \frac{d_{1}^{2}\left(q, q^{N}\right)}{2 \epsilon} \int_{0}^{t}|y(s)|_{\mathcal{V}}^{2}+\frac{\epsilon}{2} \int_{0}^{t}\left|\Delta^{N}(s)\right|_{\mathcal{V}}^{2} d s .
\end{aligned}
$$

Now, using the assumption that $\sigma(q)$ is uniformly $\mathcal{V}$-bounded for all $q \in Q$, we obtain an upper bound on the fifth term by arguing

$$
\begin{aligned}
\int_{0}^{t} \sigma\left(q^{N}\right)( & \left.(s)-P^{N} y(s), \Delta^{N}(s)\right) d s \\
\leq & \gamma_{1} \int_{0}^{t}\left|y(s)-P^{N} y(s)\right|_{\mathcal{V}}\left|\Delta^{N}(s)\right|_{\mathcal{V}} d s \\
\leq & \frac{\gamma_{1}^{2}}{2 \epsilon} \int_{0}^{t}\left|y(s)-P^{N} y(s)\right|_{\mathcal{V}}^{2} d s+\frac{\epsilon}{2} \int_{0}^{t}\left|\Delta^{N}(s)\right|_{\mathcal{V}}^{2} d s .
\end{aligned}
$$

Finally, we must obtain a bound on the nonlinear terms. We find

$$
\begin{aligned}
\int_{0}^{t}\langle g(q) & \left.(y(s))-g\left(q^{N}\right)\left(y^{N}(s)\right), \Delta^{N}(s)\right\rangle d s \\
\leq & \int_{0}^{t}\left|\left\langle g(q)(y(s))-g\left(q^{N}\right)(y(s))+g\left(q^{N}\right)(y(s))-g\left(q^{N}\right)\left(y^{N}(s)\right), \Delta^{N}(s)\right\rangle\right| d s \\
\leq & \int_{0}^{t}\left|\left\langle g(q)(y(s))-g\left(q^{N}\right)(y(s)), \Delta^{N}(s)\right\rangle\right| d s \\
& +\int_{0}^{t}\left|\left\langle g\left(q^{N}\right)(y(s))-g\left(q^{N}\right)\left(y^{N}(s)\right), \Delta^{N}(s)\right\rangle\right| d s .
\end{aligned}
$$

To treat the first term on the right side of (32), we use the Cauchy-Schwarz inequality, (9), and condition $\mathrm{C} 2$ ), which states that $g$ depends continuously on the parameter $q$, to obtain

$$
\begin{aligned}
\int_{0}^{t}\left|\left\langle g(q)(y(s))-g\left(q^{N}\right)(y(s)), \Delta^{N}(s)\right\rangle\right| d s \\
\leq \int_{0}^{t}\left|g(q)(y(s))-g\left(q^{N}\right)(y(s))\right|\left|\Delta^{N}(s)\right| d s \\
\leq \int_{0}^{t} d_{2}\left(q, q^{N}\right)|y(s)|\left|\Delta^{N}(s)\right| d s \\
\leq \frac{k^{2} d_{2}^{2}\left(q, q^{N}\right)}{2 \epsilon} \int_{0}^{t}|y(s)|_{\mathcal{V}}^{2} d s+\frac{k^{2} \epsilon}{2} \int_{0}^{t}\left|\Delta^{N}(s)\right|_{\mathcal{V}}^{2} d s .
\end{aligned}
$$


The last term in inequality (32) can be estimated by

$$
\begin{aligned}
& \int_{0}^{t}\left|\left\langle g\left(q^{N}\right)(y(s))-g\left(q^{N}\right)\left(y^{N}(s)\right), \Delta^{N}(s)\right\rangle\right| d s \\
& =\int_{0}^{t} \mid\left\langle g\left(q^{N}\right)(y(s))-g\left(q^{N}\right)\left(P^{N} y(s)\right)\right. \\
& \left.\quad+g\left(q^{N}\right)\left(P^{N} y(s)\right)-g\left(q^{N}\right)\left(y^{N}(s)\right), \Delta^{N}(s)\right\rangle \mid d s \\
& \leq \int_{0}^{t}\left|\left\langle g\left(q^{N}\right)(y(s))-g\left(q^{N}\right)\left(P^{N} y(s)\right), \Delta^{N}(s)\right\rangle\right| d s \\
& \quad+\int_{0}^{t}\left|\left\langle g\left(q^{N}\right)\left(P^{N} y(s)\right)-g\left(q^{N}\right)\left(y^{N}(s)\right), \Delta^{N}(s)\right\rangle\right| d s,
\end{aligned}
$$

and is treated as in Section 2.2.5 of [2] when proving uniqueness of solutions. That is,

$$
\begin{aligned}
& \int_{0}^{t}\left|\left\langle g\left(q^{N}\right)(y(s))-g\left(q^{N}\right)\left(P^{N} y(s)\right), \Delta^{N}(s)\right\rangle\right| d s \\
& \quad \leq \int_{0}^{t}\left|\left\langle\int_{0}^{1} g\left(q^{N}\right)^{\prime}\left(\theta y(s)+(1-\theta) P^{N} y(s)\right)\left[y(s)-P^{N} y(s)\right] d \theta, \Delta^{N}(s)\right\rangle\right| d s \\
& \quad \leq \tilde{C}_{3} \int_{0}^{t}\left|y(s)-P^{N} y(s)\right|\left|\Delta^{N}(s)\right| d s \\
& \quad \leq \frac{k^{2} \tilde{C}_{3}^{2}}{2 \epsilon} \int_{0}^{t}\left|y(s)-P^{N} y(s)\right|_{\mathcal{V}}^{2} d s+\frac{k^{2} \epsilon}{2} \int_{0}^{t}\left|\Delta^{N}(s)\right|_{\mathcal{V}}^{2} d s,
\end{aligned}
$$

where we have used (9) and condition A6). Similarly,

$$
\begin{aligned}
\int_{0}^{t} \mid\langle g & \left.\left(q^{N}\right)\left(P^{N} y(s)\right)-g\left(q^{N}\right)\left(y^{N}(s)\right), \Delta^{N}(s)\right\rangle \mid d s \\
& \leq \int_{0}^{t}\left|\left\langle\int_{0}^{1} g\left(q^{N}\right)^{\prime}\left(\theta P^{N} y(s)+(1-\theta) y^{N}(s)\right)\left[P^{N} y(s)-y^{N}(s)\right] d \theta, \Delta^{N}(s)\right\rangle\right| d s \\
& \leq \tilde{C}_{3} \int_{0}^{t}\left|P^{N} y(s)-y^{N}(s) \| \Delta^{N}(s)\right| d s \\
& =\tilde{C}_{3} \int_{0}^{t}\left|\Delta^{N}(s)\right|^{2} d s
\end{aligned}
$$

Combining (29)-(31) and (33)-(35), we obtain the inequality

$$
\begin{aligned}
\frac{1}{2}\left|\Delta^{N}(t)\right|^{2} & +\left[k_{1}-\left(3+2 k^{2}\right) \frac{\epsilon}{2}\right] \int_{0}^{t}\left|\Delta^{N}(s)\right|_{\mathcal{V}}^{2} d s \\
& \leq \frac{1}{2 \epsilon} \int_{0}^{t}\left|F\left(s ; q^{N}\right)-F(s ; q)\right|_{\mathcal{V}^{*}}^{2} d s+\frac{d_{1}^{2}\left(q, q^{N}\right)}{2 \epsilon} \int_{0}^{t} \mid\left(\left.y(s)\right|_{\mathcal{V}} ^{2} d s\right.
\end{aligned}
$$




$$
\begin{aligned}
& +\frac{\gamma_{1}^{2}}{2 \epsilon} \int_{0}^{t}\left|y(s)-P^{N} y(s)\right|_{\mathcal{V}}^{2} d s+\frac{k^{2} d_{2}^{2}\left(q, q^{N}\right)}{2 \epsilon} \int_{0}^{t}|y(s)|_{\mathcal{V}}^{2} d s \\
& +\frac{k^{2} \tilde{C}_{3}^{2}}{2 \epsilon} \int_{0}^{t}\left|y(s)-P^{N} y(s)\right|_{\mathcal{V}}^{2} d s+\tilde{C}_{3} \int_{0}^{t}\left|\Delta^{N}(s)\right|^{2} d s \\
\equiv & \delta^{N}(t)+\mu \int_{0}^{t}\left|\Delta^{N}(s)\right|^{2} d s . \\
\leq & \delta^{N}(T)+\mu \int_{0}^{t}\left|\Delta^{N}(s)\right|^{2} d s .
\end{aligned}
$$

Note that $\delta^{N}(T) \rightarrow 0$ as $N \rightarrow \infty$ by conditions B3) and C3), the assumption $q^{N} \rightarrow q$, and the result that $y \in L_{2}((0, T), \mathcal{V})$. Choosing $\epsilon>0$ so that $k_{1}-\left(3+2 k^{2}\right) \frac{\epsilon}{2}>0$, ignoring the second term on the left side of the inequality (36), and applying Gronwall's lemma, we obtain the desired result

$$
\left|\Delta^{N}(t)\right|^{2} \leq \delta^{N}(T) e^{\mu T}
$$

that is, $\Delta^{N} \rightarrow 0$ in $C([0, T], \mathcal{H})$ as $N \rightarrow \infty$. Furthermore, ignoring the first term in the left side of inequality (36) and using the result (37), we find

$$
\left|\Delta^{N}\right|_{\mathcal{V}} \rightarrow 0
$$

in $L_{2}(0, T)$ and hence almost everywhere. Taken with the remarks at the beginning of these arguments, this establishes the results of the theorem.

\subsection{Parameter Estimation for the TCDD Model}

For the model presented in Section 2.1, each of the parameters lies in some compact subset of Euclidean space, with the possible exception of the time-dependent boundary term, $A q_{2}$. Our initial efforts will involve a fixed parameterization of $A q_{2}$ represented in terms of a finite number of piecewise linear continuous basis elements, so that the problem is conceptually equivalent to the constant parameter case in that the minimization occurs over a finite parameter set [7]. In this case, the parameter estimation problem will involve the finite dimensional approximating sets $Q^{M}$. For typical examples of admissible parameter sets involving both $Q$ and $Q^{M}$, the reader is referred to [7].

We state without proof that conditions A1)-A4), A6), and C1)-C3) hold for the TCDD model. This statement follows from proofs and arguments given in [2]. For the state spaces $\mathcal{V}$ and $\mathcal{H}$, conditions B2)-B3) are satisfied for the choice of piecewise linear continuous basis elements for the finite dimensional subspace $\mathcal{H}^{N}$. Therefore, this example falls into the framework of the theoretical results presented in this section.

Finally, we note that the TCDD model includes two time delays, $\tau_{c}$ and $\tau_{r}$, with $\tau_{c} \ll \tau_{r}$. A solution to the parameter identification problem is guaranteed over each delay 
interval of length $\tau_{c}$, and is continued forward in time by the method of steps. For a complete discussion, the reader is referred to [2].

\section{Numerical Results}

In this section, we discuss our numerical results. First, we outline the techniques used in obtaining a numerical solution for the initial-boundary-value problem (1) and discuss some of the simulated solutions. Then we show how we can use the simulations for the forward problem to solve the parameter estimation (or inverse) problem described in Section 3.

\subsection{Forward Problem}

Here, we present an overview of the numerical methods used to obtain our simulations. All coefficient matrices, functions, vectors, etc. are as described in [2]. The values for the system parameters are as given in [2]; in particular, $\tau_{c}=1$ minute is the circulatory delay, $\tau_{r}=6$ hours is the induction delay, and $\mathcal{D}_{N}=5.0$ is the dispersion number, unless otherwise noted. Our numerical scheme is based on the weak formulation [4] of problem (1) described in Section 2.

For ease of notation, we define

$$
y=\left[C_{B}, C_{u_{H}}, C_{A h-T}, C_{A h}, C_{P r-T}, C_{P r}, C_{a}\right]^{T}=\left[y_{1}, \ldots, y_{7}\right]^{T} .
$$

\section{Finite Element Formulation}

Let $0=x_{0}<x_{1}<\ldots<x_{N}=1$ be a uniform partition of the interval [0,1] into $N$ subintervals of length $h=1 / N$. We take as basis elements the standard piecewise linear continuous functions, $\phi_{j}, j=0, \ldots, N$, defined by

$$
\phi_{j}(x)=\left\{\begin{aligned}
\frac{x-x_{j-1}}{h}, & x_{j-1} \leq x \leq x_{j} \\
\frac{x_{j+1}-x}{h}, & x_{j} \leq x \leq x_{j+1} \\
0, & 0 \leq x \leq x_{j-1} \text { or } x_{j+1} \leq x \leq 1
\end{aligned}\right.
$$

We define the Galerkin finite element approximations by

$$
\begin{aligned}
& y_{1}^{N}(t, x)=\sum_{j=1}^{N} \alpha_{j}^{1}(t) \phi_{j}(x), \\
& y_{i}^{N}(t, x)=\sum_{j=0}^{N} \alpha_{j}^{i}(t) \phi_{j}(x),
\end{aligned}
$$

for $i=2, \ldots, 6$, where the basis elements, $\phi_{j}$, are as described above and $y_{i}^{N}$ is the approximation for $y_{i}$. 
Next we substitute the finite element approximations (38) into the weak form of the equations. Let $y^{N}(t) \in \mathbb{R}^{N+5(N+1)+1}$ be such that

$$
y^{N}(t)=\left[\alpha^{1}(t), \alpha^{2}(t), \ldots, \alpha^{6}(t), y_{7}(t)\right]^{T}
$$

The finite dimensional system we obtain, in terms of the time-dependent coefficients of the Galerkin approximations, is given by

$$
\mathcal{M}^{N} \dot{y}^{N}(t)=\mathcal{A}^{N} y^{N}(t)+\mathcal{G}_{p}^{N}\left(y^{N}(t)\right)+\mathcal{F}^{N}(t)+\mathcal{A}_{D}^{N} y^{N}\left(t-\tau_{c}\right)+\mathcal{G}_{s}^{N}\left(y^{N}\left(t-\tau_{r}\right)\right)
$$

where the matrices $\mathcal{M}^{N}$ and $\mathcal{A}^{N}$ are elements of $\mathbb{R}^{(N+5(N+1)+1) \times(N+5(N+1)+1)}$ and the vectorvalued functions $\mathcal{G}^{N}{ }_{p}, \mathcal{F}^{N}, \mathcal{A}_{D}^{N}$, and $\mathcal{G}_{s}^{N}$ are elements of $\mathbb{R}^{N+5(N+1)+1}$ (see [2] for details).

The initial condition, $y_{0}^{N}$, for the semi-discrete problem (39) is taken as the $L_{2}$ projection of the original initial condition, $\Phi$, onto the finite element space.

\subsubsection{General Algorithm}

In this section we present an overview of the general algorithm used in our forward problem simulations. To see the effects of the protein induction delay, $\tau_{r}$, we seek solutions on the interval from zero to twenty-four hours. We have assumed that no TCDD is present in the system on the interval $[-6,0]$ hours. By making this assumption, we can ignore the induction delay term, $\mathcal{G}_{s}^{N}$, over the first induction delay interval $\left[0, \tau_{r}\right]$.

The semi-discrete problem (39) we consider is then

$$
\begin{aligned}
\mathcal{M}^{N} \dot{y}^{N}(t) & =\mathcal{A}^{N} y^{N}(t)+\mathcal{G}_{p}^{N}\left(y^{N}(t)\right)+\mathcal{F}^{N}(t)+\mathcal{A}^{N}{ }_{D} y^{N}\left(t-\tau_{c}\right)+\mathcal{G}^{N}{ }_{s}\left(y^{N}\left(t-\tau_{r}\right)\right)(40 \mathrm{a}) \\
y^{N}(s) & =y_{0}^{N}(s), \quad s \in\left[-\tau_{c}, 0\right] .
\end{aligned}
$$

The entries in the coefficient matrices $\mathcal{M}^{\mathcal{N}}$ and $\mathcal{A}^{\mathcal{N}}$, consisting of certain combinations of inner products of basis elements and their derivatives, were calculated analytically rather than using a numerical integration scheme. The nonlinear vector function $\mathcal{G}_{p}^{N}$ was treated in a similar manner. However, the nonlinear vector function $\mathcal{G}_{s}^{N}$ was calculated using the numerical integration scheme quad in MATLAB (The MathWorks, Inc., Natick, MA). In the following simulations, we take $N=16$; simulations computed with other values of $N$ are presented in [2] and suggest convergence for $N=16$.

The "method of steps" [6] for ordinary differential equations is used to computationally solve the problem (40) on successive circulatory delay intervals of length $\tau_{c}$, and again on successive induction delay intervals of $\tau_{r}$. We first find the solution over the first delay interval $\left[0, \tau_{r}\right]$, where there is no protein induction and we can ignore the induction delay term, $\mathcal{G}_{s}^{N}$. Then we find the solution over each subsequent induction delay interval; on these intervals, the protein induction has begun and we must include $\mathcal{G}_{s}^{N}$. In order to evaluate 
$y^{N}\left(t-\tau_{c}\right)$ and $y^{N}\left(t-\tau_{r}\right)$ at a particular time $t$, we store the computed solution throughout the previous delay intervals and then interpolate (assuming a variable step integration routine is used) to find the value of the solution at times $t-\tau_{c}$ and $t-\tau_{r}$. Here, for ease of computation, we assume that the final time $T$ is an integer multiple of the induction delay $\tau_{r}$ and the induction delay is an integer multiple of the circulatory delay $\tau_{c}$. More details regarding the general algorithm are given in [2].

\subsubsection{Implementation}

The computer code for the forward problem was written in MATLAB version 5.1 (The MathWorks, Inc., Natick, MA) and computations were carried out on a Sun Sparc Ultra 10 workstation. The MATLAB routine ode15s, which is a variable order, variable step method based on numerical differentiation formulas, was used for time stepping. The relative and absolute error tolerances were set to $1 \times 10^{-6}$. Since this is a variable step method, at time $t$ the solution over the previous delay interval had to be interpolated in order to determine the value of the solution at times $t-\tau_{c}$ and $t-\tau_{r}$. In order to find the value of the solution at time $t-\tau_{c}$, MATLAB's interpolation routine interp1 was used with the spline option. This method fits the data with cubic splines between data points, so that each segment of the curve fit has at least the same first and second derivatives as the ones adjoining it. The maximum order of the integrator was set to two in order for it to match the range of accuracy of both the interpolation routine and the finite element approximations. In order to find the value of the solution at time $t-\tau_{r}$, linear interpolation was used.

\subsubsection{Model Simulations}

First we focus on the solutions on the time interval from zero to twelve hours, in order to see the effect of the delayed induction of CYP1A2. In these simulations we consider a special case of the original model to facilitate the computation of simulated solutions and to make the effects of the induction delay term more pronounced. We use a special case of the boundary condition at $x=1$; we set $v=0$ and $q_{2}(t)=0$ in our original boundary condition

$$
\left.v C_{B}(t, 1)+C_{a}(t)-D \frac{\partial C_{B}}{\partial x}(t, 1)\right)=q_{2}(t)
$$

to obtain

$$
\frac{\partial C_{B}}{\partial x}(t, 1)=0
$$

This condition permits computation of simulated solutions over longer time intervals without error accumulation so that qualitative properties can be demonstrated. Since it is a special case of our original boundary condition, the theory discussed previously still holds. In addition, we set $I_{P r}$, the maximum induction rate of CYP1A2, equal to $434.6 \mathrm{nmol} / \mathrm{L} / \mathrm{hr}$ 
which is ten times the rate suggested by the literature [13]. This is done to magnify the effects of the induction delay in our computed solutions. Preliminary computational investigations on the qualitative behavior of the system without these changes are discussed in [14] and [2]. In particular, the behavior of the system on the time interval from zero to six hours and the dependence of the solution on the dispersion number, $\mathcal{D}_{N}$, and the circulatory delay, $\tau_{c}$, are presented.

During the time interval from zero to six hours (when $t<\tau_{r}$ ), the protein CYP1A2 is present only at a basal level. After six hours $\left(t=\tau_{r}\right)$ however, there is a dose-dependent induction of CYP1A2. This induction is represented in the model by the induction term $\mathcal{G}_{s}^{N}\left(y\left(t-\tau_{r}\right)\right)$. Since the model incorporates the binding of CYP1A2 with TCDD, as well as the other dynamics of TCDD interaction in the liver, we expect that the inclusion of the induction term in the model affects in a nonnegligible way the solutions of the system. We compare the simulated solutions with and without the induction term to study the nature of these effects.

First we consider the concentration of available CYP1A2 in the hepatocytes, denoted $C_{P r}$ (Figure 1). We note a sharp increase, followed by a gradual decline, in $C_{P r}$ due to the beginning of the induction of CYP1A2 and its subsequent binding with TCDD. The effect of this binding can also be seen in $C_{P r-T}$, the concentration of CYP1A2-TCDD complex in the hepatocytes (Figure 2). Here we note an initial period of adjustment to the elevated levels of CYP1A2, and then an increase in $C_{P r-T}$ due to the binding of CYP1A2 with TCDD. In addition, the binding of TCDD results in a relative decrease in the concentration of unbound TCDD in the hepatocytes, $C_{u_{H}}$ (Figure 3 ). Since there is a decrease in the concentration of unbound TCDD in the hepatocytes, unbound TCDD in the liver blood diffuses into the hepatocytes, where the CYP1A2 is produced; this results in a relative decrease of both $C_{u_{B}}$, the concentration of unbound TCDD in the liver blood, and $C_{B}$, the total concentration of TCDD in the liver blood (Figures 4 and 5). Furthermore, the induction of CYP1A2 indirectly affects the concentration of the Ah receptor, the other protein with which dioxin binds. We note that the relative decrease in $C_{u_{H}}$ causes a relative increase in $C_{A h}$, the concentration of available $\mathrm{Ah}$ receptor protein in the hepatocytes, and a relative decrease in $C_{A h-T}$, the concentration of Ah receptor-TCDD complex in the hepatocytes (Figures 6 and 7).

\subsection{Inverse Problem}

As we mentioned at the beginning of Section 3, our model contains many physiological, biological, and modelling parameters. When we computed the forward problem simulations, we set these parameters using values given in the literature. In order to use this model to simulate the disposition and elimination of TCDD in a specific animal, we must know the values for the parameters for the animal in question. Many of these values must be estimated from experimental data. In Section 3, we discussed the theory behind such a 
parameter estimation problem; here we provide numerical results for a problem of this type. For a demonstration example, we estimate the value of the axial dispersion number $\mathcal{D}_{N}$, a parameter that is not directly measurable and hence one that will not generally be available from experimental literature. We do this holding all other parameter values fixed. Since we do not have access to experimental data, we use the solution for the arterial blood concentration $C_{a}$ from the forward problem simulations as our data. This means that, in the context of Section 3, the observation operator $C$ is the dot product of the solution at time $t$ with the unit row vector in $\mathbb{R}^{1 \times(N+5(N+1)+1)}$ with a one in the last component.

\subsubsection{General Algorithm}

In this section, we define our notation and outline the general algorithm used in our simulations. Our goal is to find the value for $\mathcal{D}_{N}$ that minimizes the difference (in the least squares sense) between $C_{a}\left(t, \mathcal{D}_{N}\right)$, the $\mathcal{D}_{N}$-dependent solution from the forward problem, and our data. We let $C_{a}\left(t_{i}, \mathcal{D}_{N}\right)$ be the simulated solution of $C_{a}$ at time $t_{i}$, with $t_{i}$ in the time interval from zero to three hours. We denote the vector of data points or observations by $\hat{C}_{a}$, and we let $\left(\hat{C}_{a}\right)_{i}$, the value of $C_{a}$ observed at time $t_{i}$, be the $i$ th component of $\hat{C}_{a}$. Finally, $C_{a}^{*}$ is the simulated solution of $C_{a}$ with $\mathcal{D}_{N}=\mathcal{D}_{N}^{*}=5$.0; i.e., we take $\mathcal{D}_{N}^{*}=5.0$ as the true value in our simulated data.

The general algorithm consists of defining a least squares cost function and choosing an initial value (or values) for $\mathcal{D}_{N}$, the parameter to be estimated, which are then used in an optimization routine. Here we use a Nelder Mead optimization routine [15]. Since Nelder Mead is a simplex search algorithm, we are required to choose an initial simplex for $\mathcal{D}_{N}$, which in our case can be thought of as an ordered pair of initial values.

\subsubsection{Implementation and Data Creation}

The algorithm and inverse problem feasibility was tested with both noise free data and data containing various levels of random noise. The necessary computer code was written in MATLAB version 5.1, and the computations were carried out on a Sun Sparc Ultra 10 workstation. In the Nelder-Mead simplex search algorithm for the optimization [15], we set the termination tolerance at $10^{-4}$ when no noise was present in the data and at $10^{-3}$ in the presence of noise.

In a lab setting, we would expect to see noisy data due to such factors as measurement error, human error, and environmental effects. Since we did not have experimental data for $C_{a}$, we use three types of simulated data in our inverse problem: data without noise, data with uniformly distributed random noise, and data with normally distributed random noise. We compared our ability to estimate the value of the parameter when we added $1 \%$ relative error and $5 \%$ relative error so we could demonstrate the effect of the magnitude of the noise 
on our ability to estimate the parameter correctly. All three data sets were derived from $C_{a}^{*}(t)$, the simulated solution with the "correct" value $\mathcal{D}_{N}=\mathcal{D}_{N}^{*}=5.0$. Below we describe how the data sets were created.

The data without noise is simply the original time domain solution $C_{a}^{*}(t)$ (Figure 8). We added uniformly distributed random noise to the original time domain solution so that the "data" $\hat{C}_{a u}$ (Figures 9 and 10) was given by $\left(\hat{C}_{a u}\right)_{i}=\left(1+\epsilon_{i}\right) C_{a}^{*}\left(t_{i}\right)$. In this case, the $\epsilon_{i}$ were uniformly distributed random numbers that were appropriately scaled and shifted, depending on the magnitude of the noise, so that $\epsilon_{i} \in[-.01, .01]$ for $1 \%$ relative error and $\epsilon_{i} \in[-.05, .05]$ for $5 \%$ relative error. The random numbers were generated by the MATLAB function rand, which outputs uniformly distributed random numbers from 0 to 1 . We added normally distributed random noise to the original solution to create $\hat{C}_{a n}$ (Figures 11 and 12) in a similiar fashion, so that $\left(\hat{C}_{a n}\right)_{i}=\left(1+\epsilon_{i}\right) C_{a}^{*}\left(t_{i}\right)$. Here, the $\epsilon_{i}$ were normally distributed random numbers with mean 0 and variance 1 that were scaled accordingly, so that with $99.7 \%$ certainty $\epsilon_{i} \in[-.01, .01]$ for $1 \%$ relative error and $\epsilon_{i} \in[-.05, .05]$ for $5 \%$ relative error. The MATLAB function randn generated these normally distributed random numbers.

\subsubsection{Model Simulations}

Using the data and methods described previously, we performed numerous inverse problem calculations to investigate our ability to estimate the value of the parameter $\mathcal{D}_{N}$. We considered several different situations: using "correct" data and a range of initial conditions, using the data with uniformly distributed random noise adding $1 \%$ and $5 \%$ relative error, and using the data with normally distributed random noise adding $1 \%$ and $5 \%$ relative error.

We find that when we use the data set without noise, we are able to recover the true value of $\mathcal{D}_{N}\left(\mathcal{D}_{N}^{*}=5.0\right)$ with at least four decimal places of accuracy and obtain cost function values on the order of $10^{-5}$. This is true for initial estimates for $\mathcal{D}_{N}$ with components in the range $[4.5,5.5]$. Since there is no noise in the data, these results are what we would expect and completely typical of many inverse problem calculations using data without noise. In Table 1 we present typical findings.

\begin{tabular}{||c|c|c|c||}
\hline $\begin{array}{c}\text { Initial Simplex } \\
x_{0}\end{array}$ & $\begin{array}{c}\text { Converged Value } \\
\mathcal{D}_{N}^{-}\end{array}$ & $\begin{array}{c}\text { True Value } \\
\mathcal{D}_{N}^{*}\end{array}$ & $\begin{array}{c}\text { Cost Function } \\
J\end{array}$ \\
\hline \hline$(4.5,4.51)$ & 5.0000 & 5.0 & $4.7301 \times 10^{-5}$ \\
\hline$(5.5,5.51)$ & 5.0000 & 5.0 & $4.7301 \times 10^{-5}$ \\
\hline
\end{tabular}

Table 1: Results for data without noise.

When we use the data with noise, we find that we are still able to solve the inverse problem successfully. Even with up to fifty percent error in our initial estimate, the converged values $\overline{\mathcal{D}}_{N}$ obtained from the optimization routine are, at worst, equal to $\mathcal{D}_{N}^{*}$ with error on 
the order of $10^{-3}$ and at best, are equal $\mathcal{D}_{N}^{*}$ with at least four decimal places accuracy. The values of the cost function range from $\approx 153$ to $\approx 11,609$. Tables 2 and 3 illustrate some of these results.

\begin{tabular}{||c|c|c|c|c||}
\hline$\%$ Noise & $\begin{array}{c}\text { Initial Simplex } \\
x_{0}\end{array}$ & $\begin{array}{c}\text { Converged Value } \\
\mathcal{D}_{N}^{-}\end{array}$ & $\begin{array}{c}\text { True Value } \\
\mathcal{D}_{N}^{*}\end{array}$ & $\begin{array}{c}\text { Cost Function } \\
J\end{array}$ \\
\hline \hline $1 \%$ & $(5.0,5.01)$ & 4.9990 & 5.0 & $4.5320 \times 10^{2}$ \\
\hline $1 \%$ & $(4.5,4.51)$ & 4.9990 & 5.0 & $4.5320 \times 10^{2}$ \\
\hline $1 \%$ & $(5.5,5.51)$ & 4.9990 & 5.0 & $4.7249 \times 10^{2}$ \\
\hline $5 \%$ & $(5.0,5.01)$ & 5.0034 & 5.0 & $1.1609 \times 10^{4}$ \\
\hline $5 \%$ & $(4.5,4.51)$ & 5.0034 & 5.0 & $1.1609 \times 10^{4}$ \\
\hline $5 \%$ & $(5.5,5.51)$ & 5.0034 & 5.0 & $1.1609 \times 10^{4}$ \\
\hline
\end{tabular}

Table 2: Results for data with uniformly distributed noise.

\begin{tabular}{||c|c|c|c|c||}
\hline$\%$ Noise & $\begin{array}{c}\text { Initial Simplex } \\
x_{0}\end{array}$ & $\begin{array}{c}\text { Converged Value } \\
\overline{\mathcal{D}}_{N}\end{array}$ & $\begin{array}{c}\text { True Value } \\
\mathcal{D}_{N}^{*}\end{array}$ & $\begin{array}{c}\text { Cost Function } \\
J\end{array}$ \\
\hline \hline $1 \%$ & $(5.0,5.01)$ & 5.0000 & 5.0 & $1.5342 \times 10^{2}$ \\
\hline $1 \%$ & $(4.5,4.51)$ & 5.0000 & 5.0 & $1.5342 \times 10^{2}$ \\
\hline $1 \%$ & $(5.5,5.51)$ & 5.0000 & 5.0 & $1.5342 \times 10^{2}$ \\
\hline $1 \%$ & $(2.5,2.51)$ & 5.0000 & 5.0 & $1.5342 \times 10^{2}$ \\
\hline $1 \%$ & $(7.5,7.51)$ & 5.0000 & 5.0 & $1.5342 \times 10^{2}$ \\
\hline $5 \%$ & $(5.0,5.01)$ & 4.9998 & 5.0 & $3.8822 \times 10^{3}$ \\
\hline $5 \%$ & $(4.5,4.51)$ & 4.9998 & 5.0 & $3.8822 \times 10^{3}$ \\
\hline $5 \%$ & $(5.5,5.51)$ & 4.9998 & 5.0 & $3.8822 \times 10^{3}$ \\
\hline
\end{tabular}

Table 3: Results for data with normally distributed noise.

We find that we do a better job of recovering the true value $\mathcal{D}_{N}^{*}$ when there is normally distributed noise than when there is uniformly distributed noise, no matter what the percent error. This is to be expected since the noise in the data is more likely to be "larger" with the uniformly distributed noise. To see this more clearly, we can look at $\epsilon_{i}$ for any $i$, which we described in the previous section when we discussed the creation of the data. We consider the case with $1 \%$ relative error. For the uniformly distributed noise, it is equally likely that $\epsilon_{i}$ is any number in [-.01, .01]. However, for the normally distributed noise, there is a $68 \%$ chance that the random number generated by the routine is within one standard deviation (in our case $\sigma=1$ ) from zero and a $99.7 \%$ chance that it is within three standard deviations from zero. Here, that means that there is a $99.7 \%$ chance that $\epsilon_{i} \in[-.01, .01]$ and a $68 \%$ chance that $\epsilon_{i} \in[-.0033, .0033]$. So, it is likely that the normally distributed noise has a smaller magnitude than the uniformly distributed noise. 


\section{Concluding Remarks}

The previous discussions provide a rigorous foundation for computational methods to investigate both forward simulations and inverse problems for nonlinear parabolic partial differential systems with delays. In particular, the motivating TCDD spatially distributed liver model can be treated using the computational framework presented here. The sample

computational results for this model demonstrate the applicability of our approach in the investigation of both qualitative and quantitative properties of such models. 


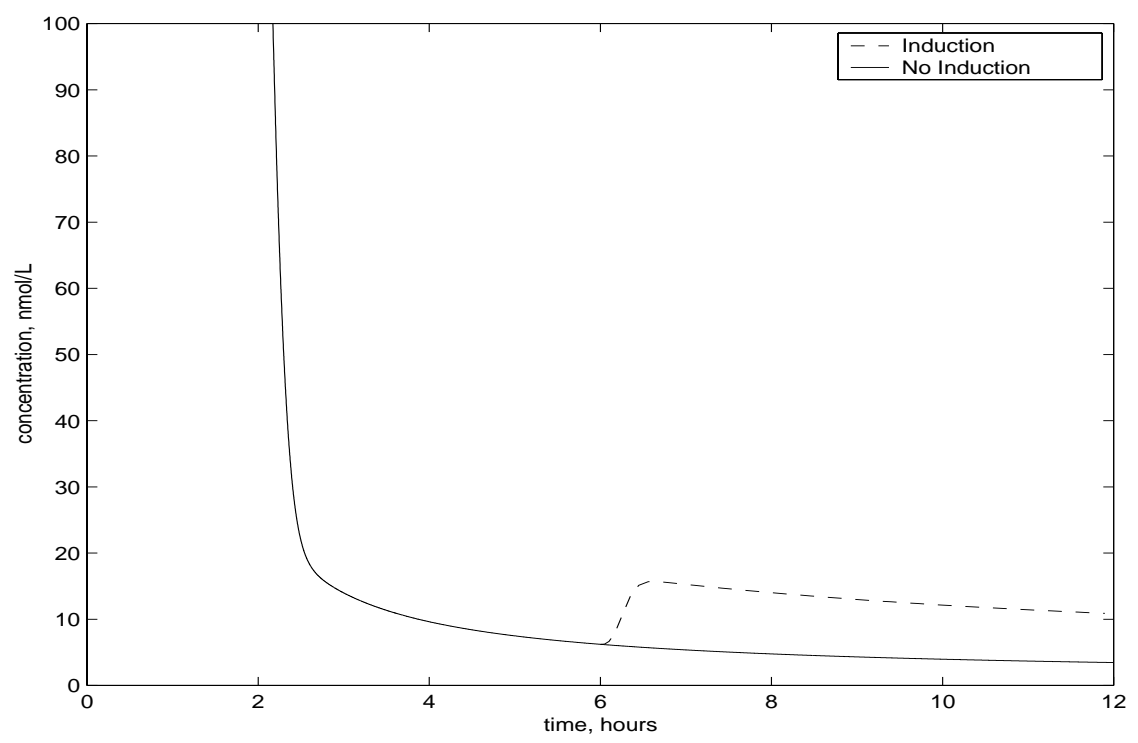

Figure 1: The effect of the induction delay on the concentration of available CYP1A2 in the hepatocytes.

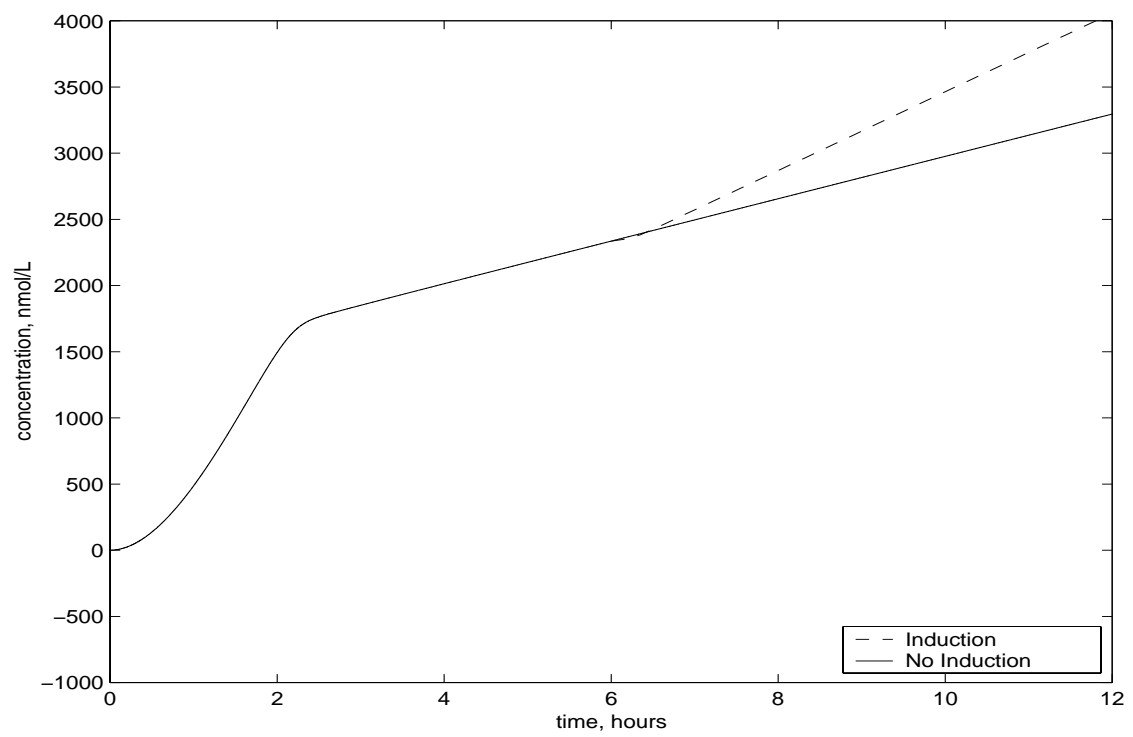

Figure 2: The effect of the induction delay on the concentration of CYP1A2-TCDD complex in the hepatocytes. 


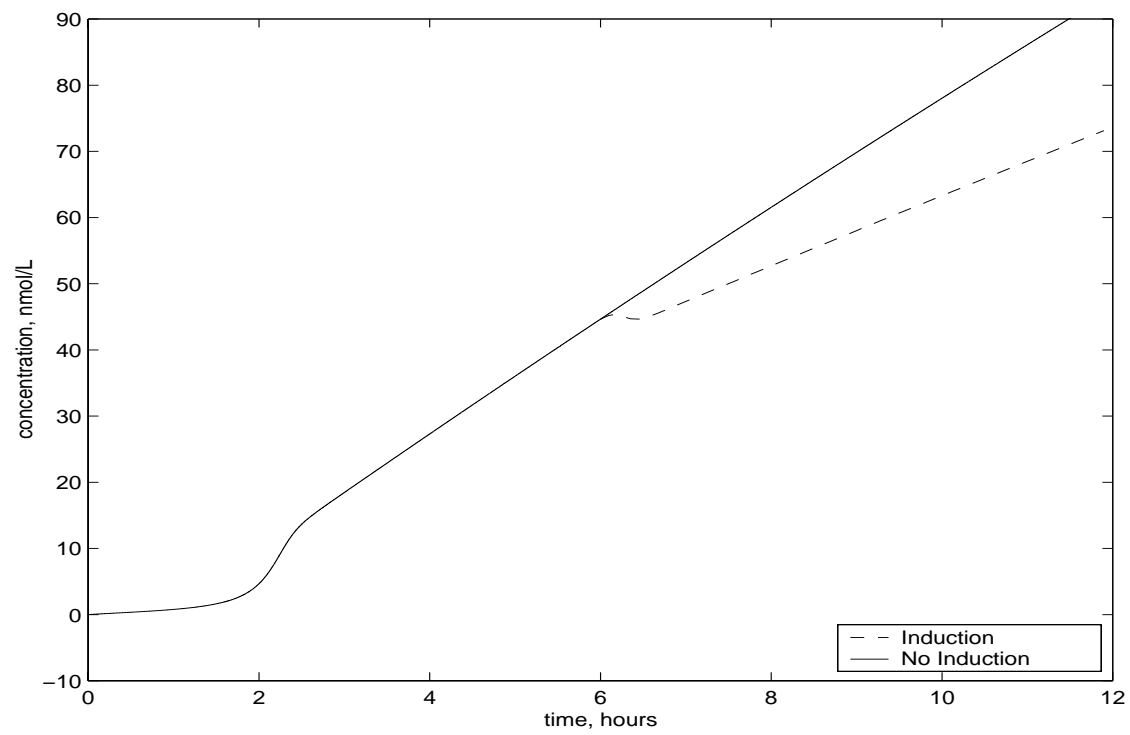

Figure 3: The effect of the induction delay on the concentration of unbound TCDD in the hepatocytes.

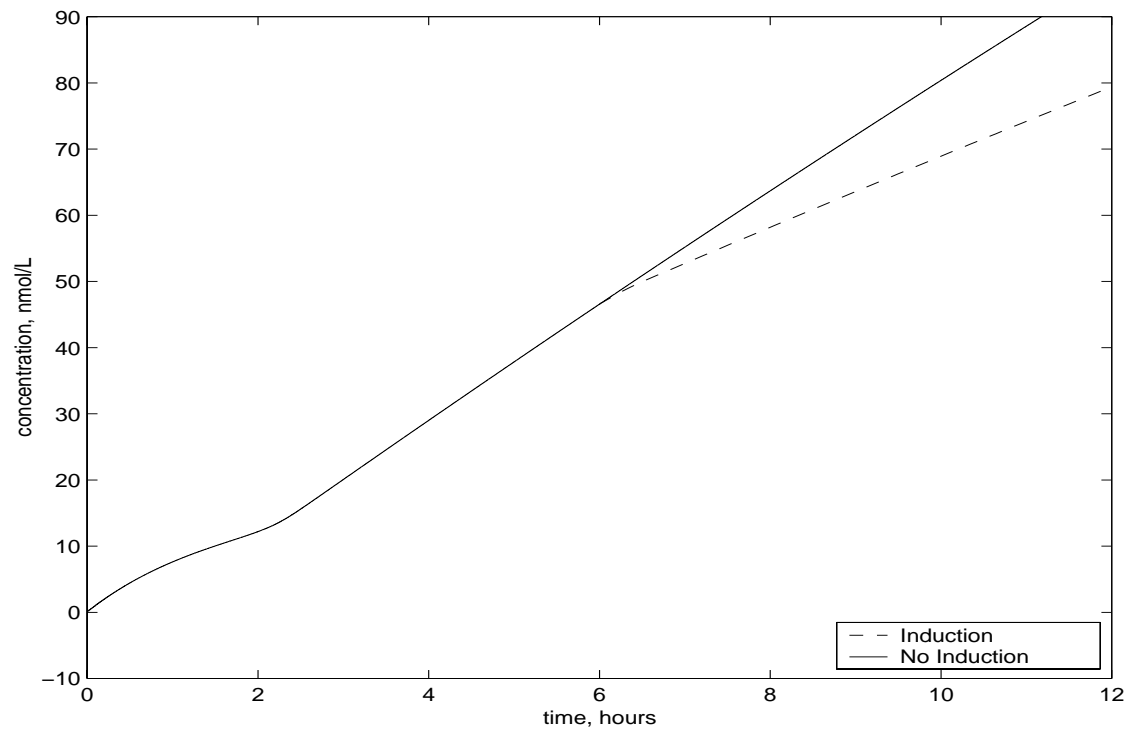

Figure 4: The effect of the induction delay on the concentration of unbound TCDD in the liver blood. 


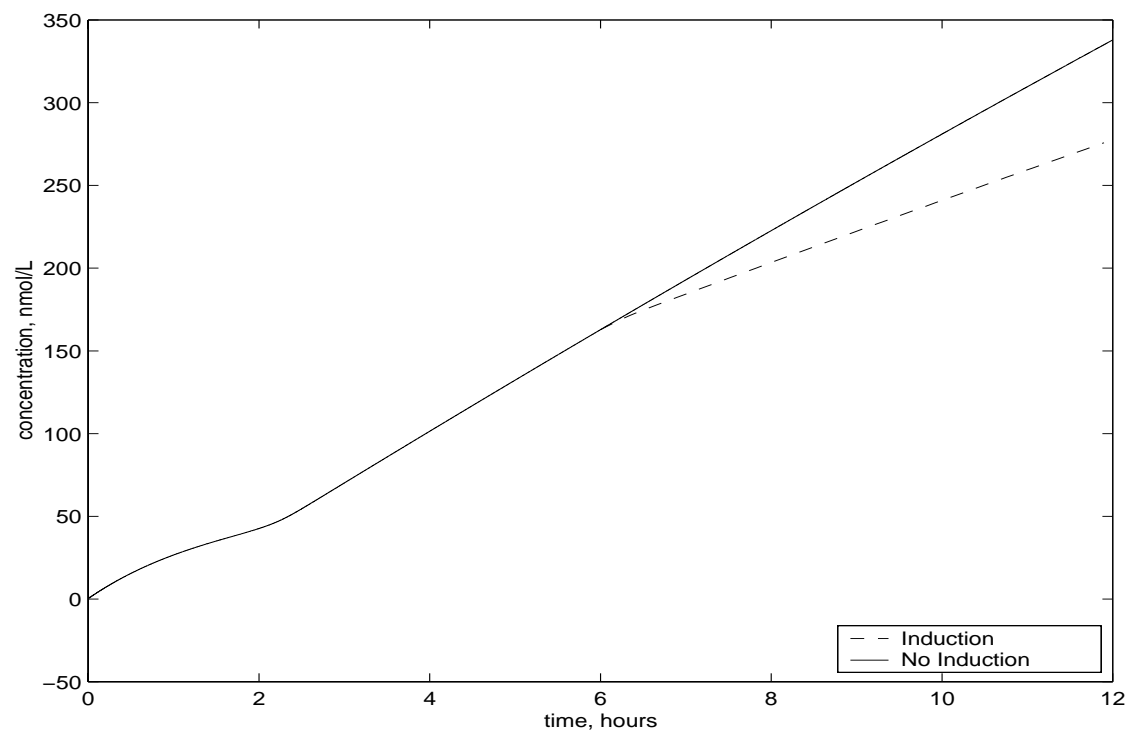

Figure 5: The effect of the induction delay on the total concentration of TCDD in the liver blood.

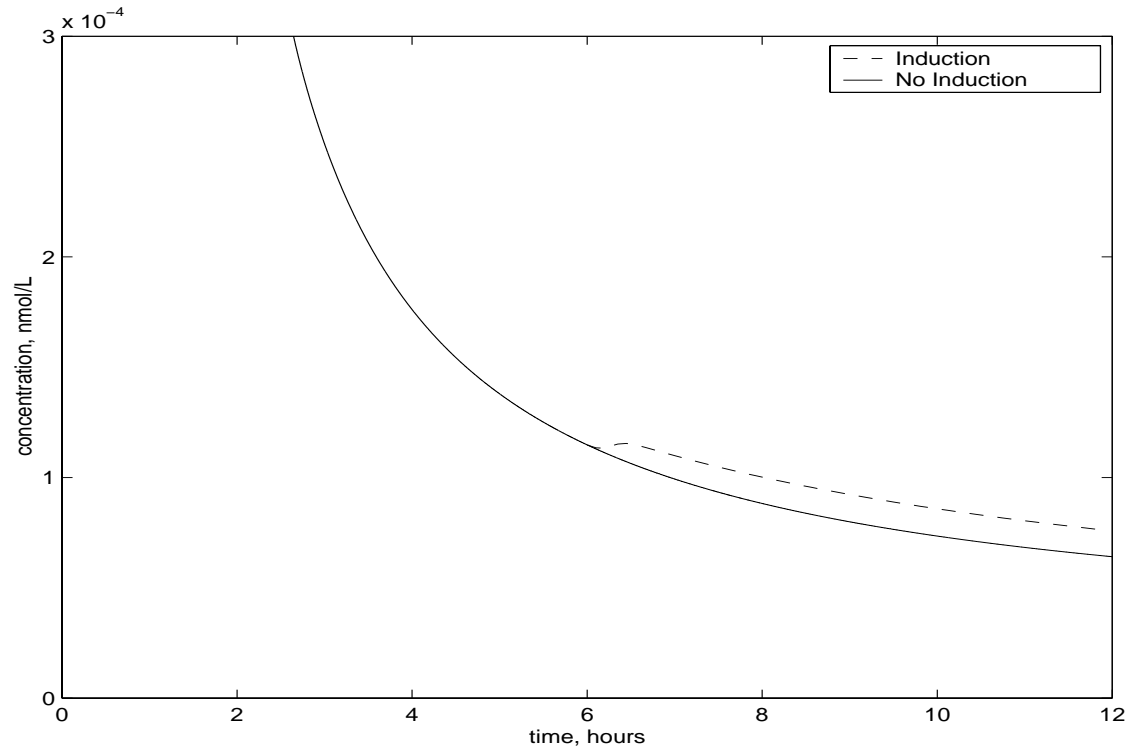

Figure 6: The effect of the induction delay on the concentration of available Ah receptor in the hepatocytes. 


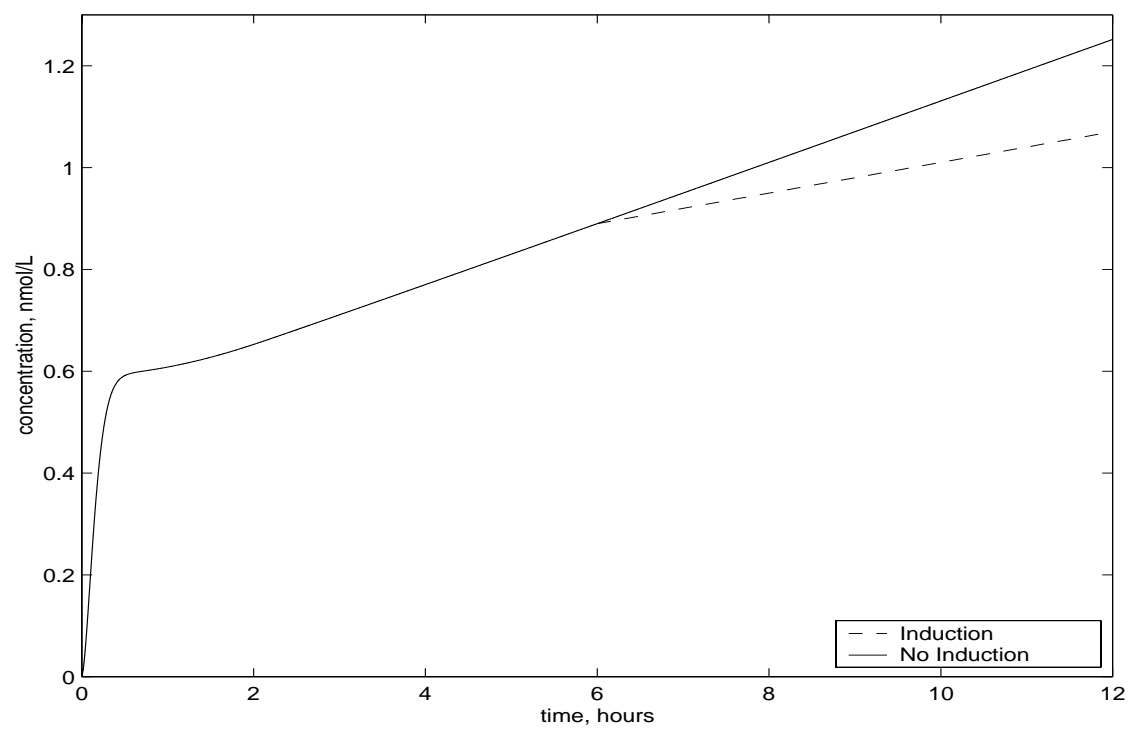

Figure 7: The effect of the induction delay on the concentration of Ah receptor-TCDD complex in the hepatocytes.

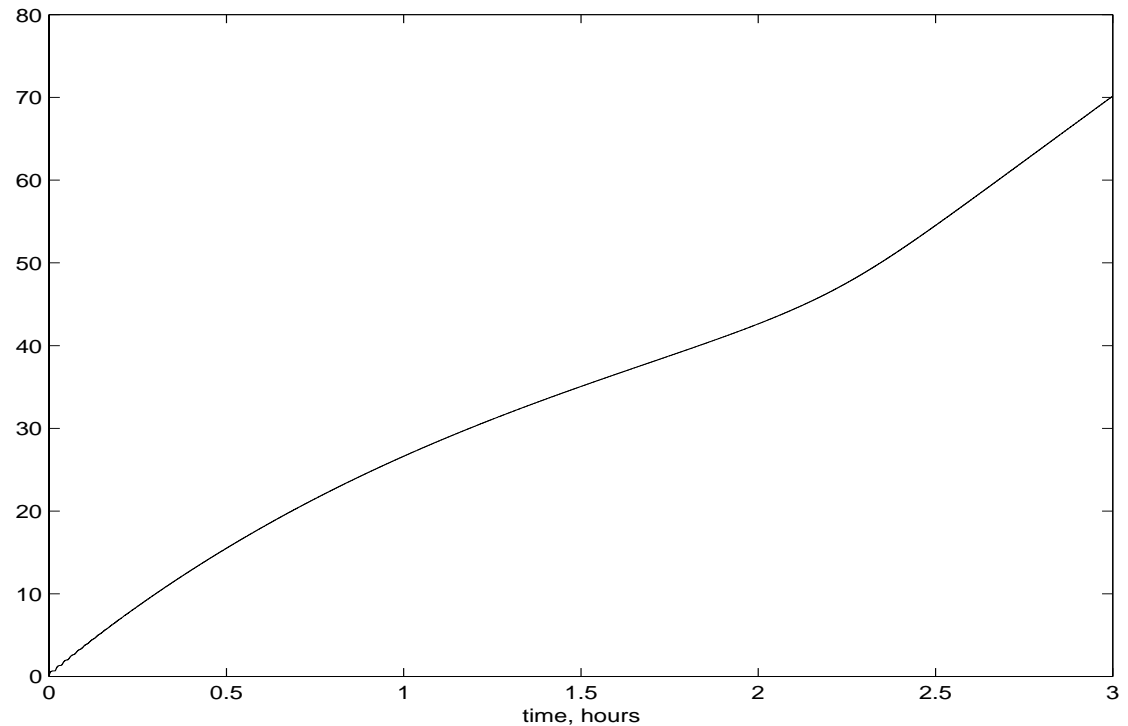

Figure 8: The computed solution for the concentration of TCDD in the arterial/venous blood compartment $\left(C_{a}\right)$ without noise. 


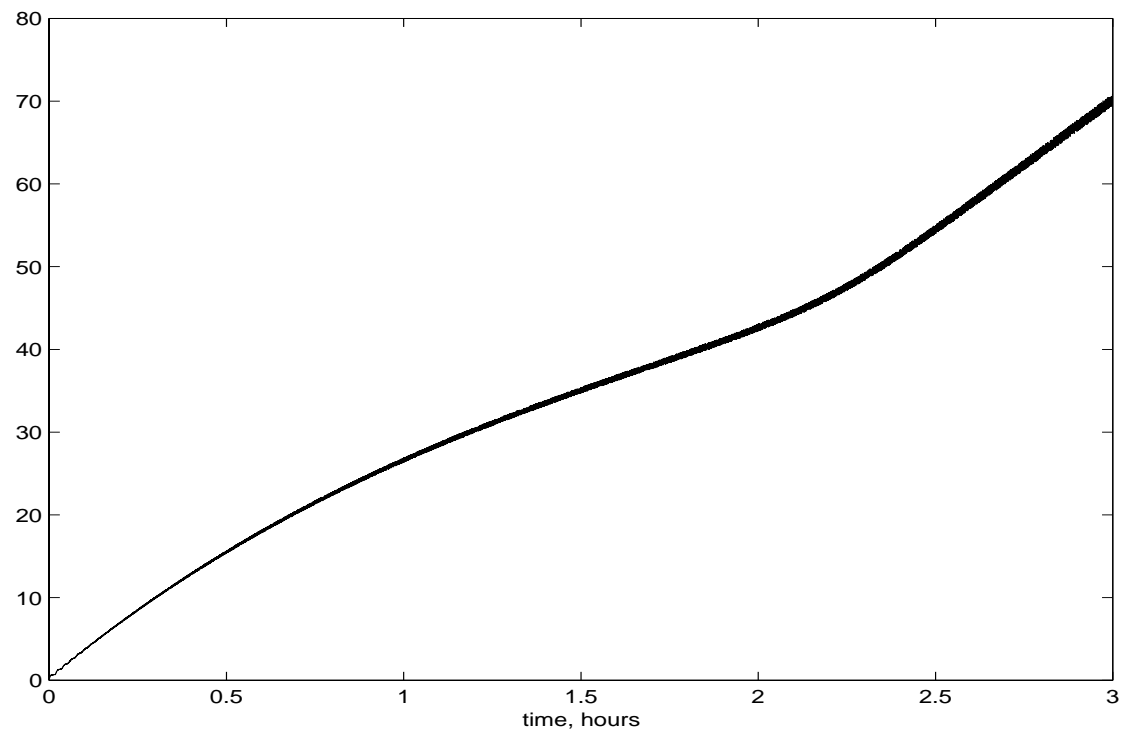

Figure 9: The computed solution for the concentration of TCDD in the arterial/venous blood compartment $\left(C_{a}\right)$ with $1 \%$ uniformly distributed random noise.

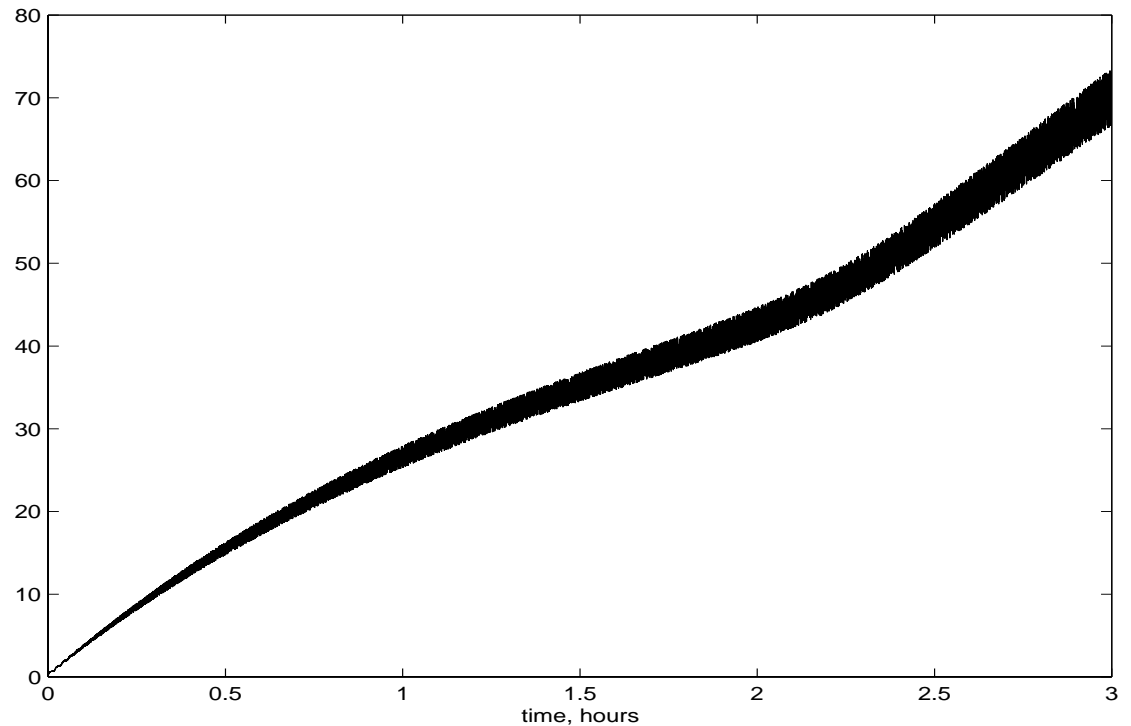

Figure 10: The computed solution for the concentration of TCDD in the arterial/venous blood compartment $\left(C_{a}\right)$ with $5 \%$ uniformly distributed random noise. 


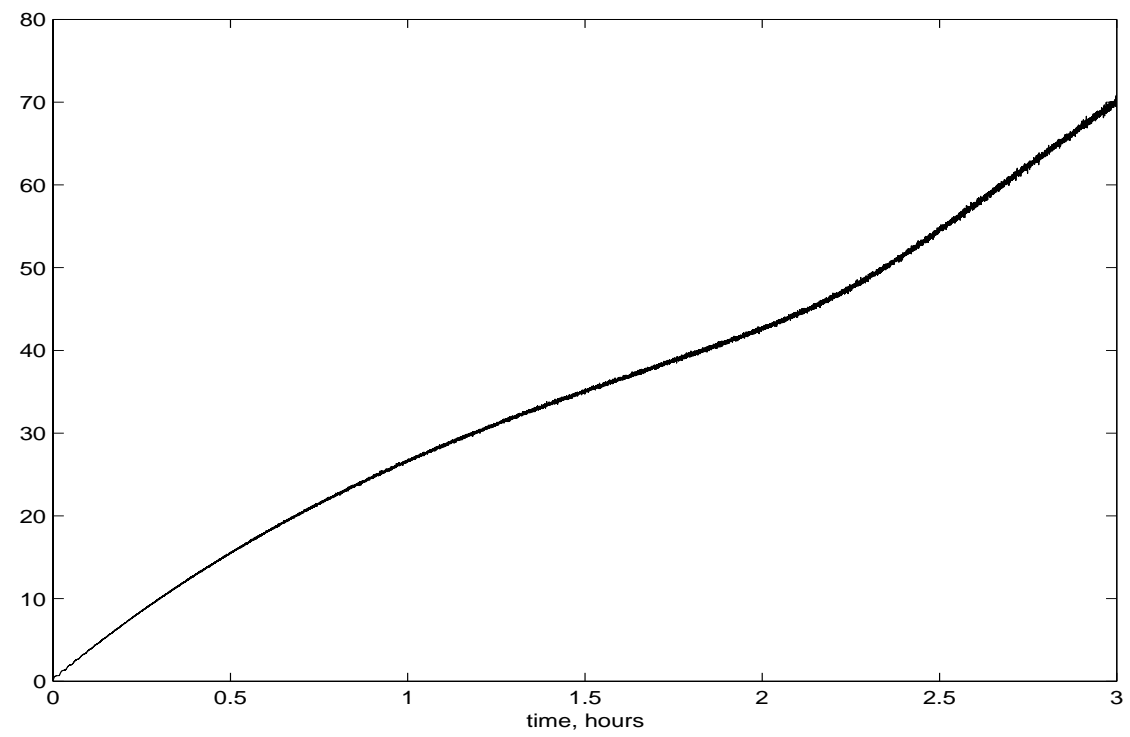

Figure 11: The computed solution for the concentration of TCDD in the arterial/venous blood compartment $\left(C_{a}\right)$ with $1 \%$ normally distributed random noise $(99.7 \%$ certainty).

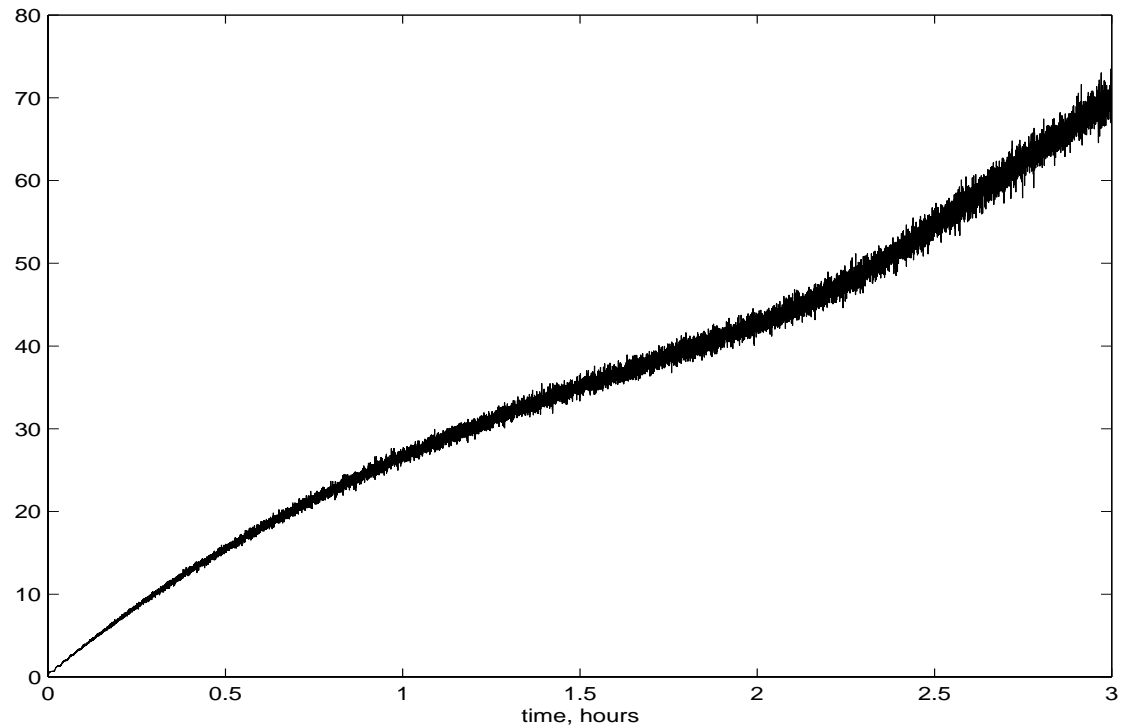

Figure 12: The computed solution for the concentration of TCDD in the arterial/venous blood compartment $\left(C_{a}\right)$ with $5 \%$ normally distributed random noise $(99.7 \%$ certainty). 


\section{References}

[1] H. T. Banks, C. J. Musante, and H. T. Tran. A dispersion model for the hepatic uptake and elimination of 2,3,7,8-tetrachlorodibenzo-p-dioxin. CRSC-TR97-29, September, 1997, Mathematical and Computer Modelling, 28:9-29, 1998.

[2] C. J. Musante. A Distributed Parameter Model to Describe the Hepatic Processing of 2,3,7,8-Tetrachlorodibenzo-p-dioxin. PhD thesis, North Carolina State University, Raleigh, NC, August 1998.

[3] Michael S. Roberts and Malcolm Rowland. A dispersion model of hepatic elimination: 1. Formulation of the model and bolus considerations. Journal of Pharmacokinetics and Biopharmaceutics, 14(3):227-260, 1986.

[4] H. T. Banks and C. J. Musante. Well-posedness for a class of abstract nonlinear parabolic systems with time delay. CRSC-TR97-30, September 1997, Nonlinear Analysis: Theory, Methods, and Applications, 35:629-648, 1999.

[5] H. T. Banks, D. S. Gilliam, and V. L. Shubov. Global solvability for damped abstract nonlinear hyperbolic systems. Differential and Integral Equations, 10:309-332, 1997.

[6] R. Bellman and K. L. Cooke. Differential-Difference Equations. Rand Corporation, 1963.

[7] H. T. Banks and K. Kunisch. Estimation Techniques for Distributed Parameter Systems. Birkhauser, Boston, 1989.

[8] H. T. Banks and Gabriella A. Pinter. Approximation results for parameter estimation in nonlinear elastomers. CRSC-TR96-34, December 1996; Control and Estimation of Distributed Parameter Series Birkhauser Intl. Series of Numerical Methods, 126:1-13, 1998.

[9] H. T. Banks, R. C. Smith, and Y. Wang. Smart Material Structures: Modeling Estimation, and Control. Masson/J. Wiley \& Sons, Paris/Chichester, 1996.

[10] H. T. Banks and K. Ito. A unified framework for approximation in inverse problems for distributed parameter systems. Control: Theory and Advanced Technology, 4(1):73-90, 1988.

[11] J. Wloka. Partial Differential Equations. Cambridge University Press, 1992.

[12] David A. Sanchez. Ordinary Differential Equations and Stability Theory: An Introduction. W. H. Freeman and Company, San Francisco, 1968. 
[13] Melvin E. Andersen, Linda S. Birnbaum, Hugh A. Barton, and Christopher R. Ecklund. Regional hepatic CYP1A1 and CYP1A2 induction with 2,3,7,8-tetrachlorodibenzo-pdioxin evaluated with a multicompartment geometric model of hepatic zonation. Toxicology and Applied Pharmacology, 144:145-155, 1997.

[14] H. T. Banks, C. J. Musante, and J. K. Raye. Predictions for a distributed parameter model describing the hepatic processing of 2,3,7,8-tetrachlorodibenzo- $p$-dioxin. $C R S C$ TR98-38, November 1998; Mathematical and Computer Modelling, to appear.

[15] C. T. Kelley. Iterative Methods for Optimization. SIAM, Philadelphia, 1999. 